\title{
Sistemas Impulsivos do Ponto de Vista das Equações Diferenciais em Medida*
}

\author{
Miguel Vinicius Santini Frasson
}

Orientador: Prof. Dr. Plácido Zoega Táboas

Dissertação apresentada ao Instituto de Ciências Matemáticas e de Computação - ICMC-USP, como parte dos requisitos para obtenção do título de Mestre em Ciências - Área: Matemática.

USP - São Carlos

Maio de 2000

* Trabalho realizado com o apoio da FAPESP 


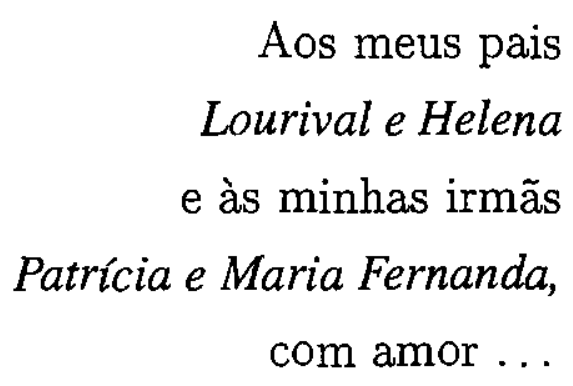




\section{Agradecimentos}

Não posso deixar de agradecer àqueles que me ajudaram a escrever as páginas dessa conquista. Os nomes citados estão ordenados alfabeticamente.

Agradeço de maneira mais especial a meus pais Helena e Lourival (Brasa), pelo amor e educação que me deram (vocês são maravilhosos). Às minhas queridas irmãs Fernanda e Patrícia, pela mais fiel amizade (amo vocês). À minha família. À Marilia Juliana, minha namorada, e à sua alegre família de Goiânia, pelo carinho e apoio nesses últimos momentos.

Aos alunos do ICMC pela amizade, e de maneira especial a Andréa, Claudemir (Caniz), Daniela, Kátia, Luciene, José (quem me dera...), Marcello (meu amigo Romário), Maria Alice, Marta, Silvia e Vera. Aos meus (mais amigos que) professores, por modelarem minha formação, em especial ao Gaspar (obrigado pela ajuda), Ires, Maria Aparecida (Cidinha), Oziride (Dide) e Valdir. Aos funcionários do ICMC.

Aos meus amigos, distantes mas não esquecidos, e em especial (em Adamantina) ao Geraldo, Wellington (Cecotte), Nilton (Nirtão) e Sérgio; e Hamilton (Ovo), de Araçatuba. A esses, obrigado pela amizade e torcida. Aos meus amigos que comigo moram Anderson, Claudemir, José, Lucas (bixo) e moraram André (Naga); e ao pessoal do Olimpo: Adriano (Aranha), Alan (Onze), Alfraino (Cabelo), Antônio (Busca Pé), Hoel (Bola), Moacyr (Cirrose), Renato (Gorpo), Ricardo (Patolino), Rodrigo (C-Bozo), Rogério (Bléia) e Samuel (Vera Verão).

Ao meu querido orientador Plácido Zoega Táboas, por todos esses anos de orientação e cuidado. Muito obrigado por tudo. 


\section{Resumo}

Estamos interessados em Equações Diferenciais Ordinárias com impulsos em momentos pré-fixados, ou seja, EDO's cujas soluções experimentam descontinuidades do primeiro tipo ao longo de uma seqüência de instantes $t_{1}<t_{2}<\cdots$. Descrevemos um contexto conveniente para o tratamento dessas equações, introduzindo resultados básicos da teoria das Equações Diferenciais em Medida. 


\section{Abstract}

We are concerned with Impulsive Differential Equations with pre-assigned moments of impulse, i.e., ODE's whose solutions undergo discontinuities of the first kind along a sequence $t_{1}<t_{2}<\cdots$ of instants. We describe a convenient setting to treat these equations by introducing basic results from the theory of Measure Differential Equations. 


\section{Sumário}

Introdução

1 Pré-requisitos 3

1 Medidas . . . . . . . . . . . . . . . . . . . 4

1.1 Medida de Lebesgue . . . . . . . . . . . . . . . . . . 6

1.2 Medidas com Sinal . . . . . . . . . . . . . . 8

1.3 Medidas Geradas por Funções . . . . . . . . . . . . . . . . . . . . . . . . . . . . . . . . . . .

2 Integração . . . . . . . . . . . . . . . . . 11

2.1 Integral de Riemann-Stieltjes . . . . . . . . . . . . . . . . 11

2.2 Integral de Lebesgue-Stieltjes . . . . . . . . . . . . . . . . 15

3 Diferenciabilidade . . . . . . . . . . . . . . . . 20

4 Funções de Variação limitada . . . . . . . . . . . . . . . . . . . . 21

4.1 Princípio da Seleção de Helly . . . . . . . . . . . . . . . . . . . . . . . . . . . . . 23

5 Continuidade Absoluta . . . . . . . . . . . . . . . . 26

6 Funções do Tipo $\varphi \ldots \ldots \ldots \ldots \ldots \ldots$

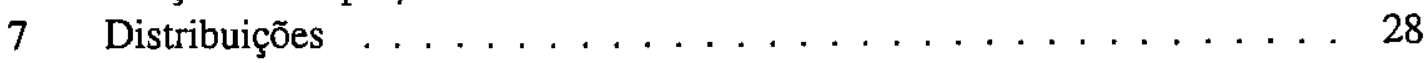

7.1 Derivada Distribucional ............. 30

2 Existência e Unicidade 33

1 Equações Diferenciais em Medida . . . . . . . . . . . . . . . . . . . . 33

2 Representação Integral . . . . . . . . . . . . . . . . . . . . . . . . . . . . . . . .

3 Existência de Soluções . . . . . . . . . . . . . . . . . . . . . . . . . . . . . . . . . 47

4 Unicidade . . . . . . . . . . . . . . . . . . . 40

3 Sistemas Lineares Impulsivos $\quad \mathbf{4 7}$

1 Sistemas Lineares Impulsivos . . . . . . . . . . . . . . . . . . . . . . . . . . . 48

2 Fórmula da Variação das Constantes . . . . . . . . . . . . . . 53

$\begin{array}{ll}\text { Bibliografia } & 57\end{array}$

Índice Remissivo $\quad 59$ 


\section{Introdução}

Muitos processos de evolução são caracterizados pelo fato de, em certos momentos, experimentarem uma mudança brusca de estado. Tais processos estão sujeitos a perturbações cuja duração é desprezível. É, portanto, natural considerarmos essas perturbações como instantâneas, ou seja, como impulsos.

Consideremos, como exemplo, um modelo simples sobre a ação de um certo medicamento no corpo humano. Assumindo que a droga é ministrada oralmente, ela é gradativamente absorvida pelo sangue no sistema gastro-intestinal e finalmente eliminada pelos rins. Sejam $x(t)$ e $y(t)$ as quantidades de droga no sistema gastrointestinal e no sangue, respectivamente, no instante $t$. Se $k_{1}$ e $k_{2}$ são taxas de absorção e eliminação, respectivamente, podemos modelar este processo por

$$
\dot{x}=-k_{1} x, \quad \dot{y}=-k_{2} y+k_{1} x .
$$

Impondo que o medicamento é ministrado nos instantes $0, t_{1}, t_{2}, \ldots, t_{n}$ nas quantidades $a_{0}, a_{1}, a_{2}, \ldots, a_{n}$, as soluções devem satisfazer

$$
\begin{aligned}
& x\left(t_{i}+\right)=x\left(t_{i}-\right)+a_{i}, \\
& y\left(t_{i}+\right)=y\left(t_{i}-\right), \\
& x(0)=a_{0}, \quad y(0)=0 .
\end{aligned}
$$

O sistema ( $\star$, , sujeito a estas condições, define um sistema impulsivo.

Para a eficácia do tratamento, é necessário que a quantidade $y(t)$ de droga no sangue permaneça em um certo patamar. Esta situação mostra a relevância de estudarmos equações diferenciais que envolvam impulsos.

As Equações Diferenciais em Medida constituem um contexto natural para o estudo das equações diferenciais ordinárias sujeitas a impulsos em tempos pré-fixados, 
impulsos estes que podem depender do tempo e dos limites laterais da solução nos instantes de salto. Este trabalho é um estudo de parte da teoria básica dessas equações, tais como problemas de existência e unicidade de soluções, equações cujos impulsos dependem linearmente da solução e alguns resultados gerais, como extensões de resultados da teoria das equações ordinárias para equações em medida. 


\section{Capítulo 1}

\section{Pré-requisitos}

Este capítulo tem por finalidade apresentar alguns resultados já conhecidos que serão utilizados nos próximos capítulos, nos quais estudaremos as equações diferenciais em medida (que abreviamos por EDM). Essas equações admitem funções descontínuas como soluções, necessitando portanto de uma estrutura mais geral na qual tais funções possuem derivadas, papel este cumprido pelas distribuições. Proporemos uma representação integral para as EDM (ver Seção 2 do Capítulo 2) que nos possibilitará remeter os problemas para a teoria de integração. Para tanto, devemos nos preocupar com certas classes de funções e suas propriedades.

Uma distribuição é um funcional linear contínuo no espaço das funções de suporte compacto. No espaço das distribuiçōes, definimos a derivada distribucional. Funções pertencentes a certas classes podem ser identificadas naturalmente a distribuições. Nestes casos, uma função pode possuir dois tipos de derivada: a usual e a distribucional. Veremos que essas derivadas coincidem para tais funções. Uma função de variação limitada possui derivada em quase toda parte ${ }^{1}$. Além disso, se for contínua à direita, gerará uma medida (com sinal) de Borel. Continuidade absoluta garante, além da continuidade, boa parte da estrutura de diferenciabilidade, no sentido que $f$ tem derivada definida em quase toda parte e $f(t)=f(a)+\int_{a}^{t} f^{\prime}(s) d s$, caso $f$ seja absolutamente contínua.

Parte deste capítulo está contida no livro de Royden [6]. O capítulo sobre preliminares do livro de Pandit-Deo [5] também foi bastante utilizado. Alguns tópicos

\footnotetext{
'Uma propriedade é dita valer em quase toda parte ou quase sempre se for falsa apenas dentro de um conjunto de medida nula.
} 
sobre funções de variação limitada e integração de Riemann-Stieltjes foram retirados de Rudin [7]. Os tópicos sobre distribuições foram extraídos de Pandit-Deo [5] e Hounie [3].

Por $\left(f_{n}\right)$ denotamos as seqüências $f_{n}, \operatorname{com} n \in \mathbb{N} .\left\{E_{\lambda}\right\}_{\lambda \in \Lambda}$ denota as famílias ou coleções, de conjuntos ou funções. $f\left(x_{0}+\right)$ denota $\lim f(x)$ quando $x \rightarrow x_{0}+$, o limite lateral à direita. Analogamente, $f\left(x_{0}-\right)=\lim f(x)$ quando $x \rightarrow x_{0}-$. Os símbolos

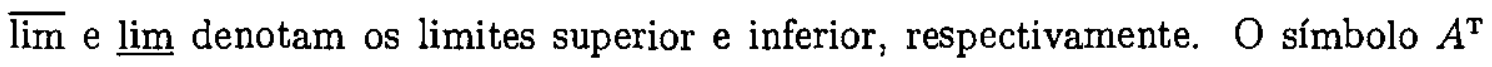
denota a transposta da matriz $A$. Por $\overline{\mathbb{R}}$ entendemos o sistema de números reais estendidos, isto é, $\mathbb{R} \cup\{+\infty,-\infty\},-\infty<x<\infty, \forall x \in \mathbb{R}$, com as operações induzidas pelo processo de limite, ou seja, se $a$ e $b$ são números reais estendidos e $\diamond$ é uma operação em $\mathbb{R}$, definimos $a \diamond b:=\lim _{x \rightarrow a} \lim _{y \rightarrow b}(x \diamond y), x, y \in \mathbb{R}$, se o limite resultar em um elemento de $\overline{\mathbb{R}}$. Desse modo, $\infty-\infty$ e $\infty / \infty$ não estão definidos.

\section{Medidas}

Inicialmente faremos algumas considerações preliminares superficiais sobre conceitos que trataremos mais detidamente a seguir.

O conceito de medida surge com o comprimento de um intervalo na reta, que é a diferença entre os extremos superior e inferior. A função "comprimento" está definida no conjunto dos intervalos de $\mathbb{R}$, atribuindo um número real estendido $l(I)$ a cada intervalo $I$. No entanto, gostaríamos de atribuir uma medida a conjuntos mais complicados que intervalos. Poderíamos definir a medida de um aberto como a soma dos comprimentos dos intervalos abertos que o constitui. Nesta direção, surgiu a Teoria da Medida, com a medida de Lebesgue sobre os conjuntos mensuráveis, e também uma ampla fundamentaçāo para teorias sobre integração. Com o tempo, apareceram novas idéias sobre outras maneiras de se medir conjuntos, na teoria de medida geral, que toma algumas propriedades da medida de Lebesgue como axiomas è constrói uma teoria mais geral, admitindo números negativos ou complexos como medida de um conjunto. Também, pensou-se em como alterar a "densidade" da medida nas regiōes da reta real, controlando esta densidade com as "funções peso", nas integrais de Stieltjes.

Nesta seção veremos algumas definições e resultados sobre a teoria de medida, 
mas este texto não pretende se alongar nas demonstrações técnicas desta teoria. Descreveremos quais são os conjuntos Lebesgue-mensuráveis e as propriedades da medida de Lebesgue, como a de ser uma medida completa, por exemplo. Os resultados sobre medidas com sinal, medidas geradas por funções e integração serão necessários quando indicarmos como as funções contínuas à direita de variação limitada podem gerar medidas, e como são feitas as identificações entre estas funções e suas distribuições.

Definição 1.1 ( $\sigma$-álgebra). Uma família $\mathcal{A}$ de subconjuntos de um conjunto $X$ é dita uma $\sigma$-álgebra quando

i. $\varnothing, X$ pertencem a $\mathcal{A}$;

ii. Se A pertence a $\mathcal{A}$, entäo o conjunto complementar de $A$, denotado por $A^{\mathrm{c}}$, pertence a $\mathcal{A}$;

iii. Se $\left\{A_{i}\right\}_{i \in \mathbb{N}}$ é uma familia de conjuntos em $\mathcal{A}$, então a uniāo $\bigcup_{i=1}^{\infty} A_{i}$ pertence a $\mathcal{A}$.

Definição 1.2 (Medida). Uma medida $\mu$ definida em uma $\sigma$-álgebra $\mathcal{A}$ é uma função real estendida não negativa satisfazendo as condições

i. $\mu(\varnothing)=0$

ii. Para toda família disjunta $\left\{X_{i}\right\}_{i \in \mathbb{N}}$ em $\mathcal{A}$, vale

$$
\mu\left(\bigcup_{i=1}^{\infty} X_{i}\right)=\sum_{i=1}^{\infty} \mu\left(X_{i}\right)
$$

A segunda condição é conhecida como $\sigma$-aditividade. Os elementos de uma $\sigma$ álgebra sāo chamados conjuntos mensuráveis (ou $\mu$-mensuráveis). Se $\mu X<\infty$, então $\mu$ é dita finita. Se todo subconjunto $\tilde{A}$ de um conjunto mensurável $A$ de medida nula $(\mu A=0)$ é também mensurável, $\mu$ é dita uma medida completa. Veremos que a medida de Lebesgue, definida em $\mathfrak{M}$, é completa (veja seção 1.1). A medida de Lebesgue definida nos conjuntos mensuráveis de $X=[0,1]$ é finita. O par $(X, \mathcal{A})$ é chamado um espaço mensurável e, a um espaço mensurável dotado de uma medida $\mu$, chamamos um espaço de medida, denotando $(X, \mathcal{A}, \mu)$. Uma medida $\mu$, como na definição anterior, também é chamada uma medida positiva, por $\mu$ ser não negativa. Trataremos aqui as medidas no sentido da definição 1.2 por esta segunda 
denominação, usando o termo "medida", mais geral, também para as medidas com sinal (ver definição 1.11). Uma importante propriedade de uma medida $\mu$ é dada pela proposição seguinte.

Proposição 1.3. Seja $\left(A_{i}\right)$ uma sequência decrescente de conjuntos $\mu$-mensuráveis, ou seja, $A_{n+1} \subseteq A_{n}$ para cada n. Se $\mu A_{1}<\infty$, então

$$
\mu\left(\bigcap_{i=1}^{\infty} A_{i}\right)=\lim _{n \rightarrow \infty} \mu A_{n} .
$$

Sua demonstração pode ser encontrada em Royden [6], Proposição 11.2.

\subsection{Medida de Lebesgue}

A idéia da medida de Lebesgue, grosso modo, é medir um intervalo $I$ por seu comprimento $l(I)$, e outros subconjuntos de $\mathbb{R}$ por algo como a "soma dos comprimentos dos intervalos que o compõem". Esta característica permitirá à medida de Lebesgue ser invariante por translações, ou seja, $m(A)=m(A+x)$, em que $A+x$ é o conjunto $\{y+x \mid y \in A\}$, independentemente do valor de $x \in \mathbb{R}$.

Definição 1.4 (Medida Exterior). Seja A um conjunto de números reais. A quantidade $m^{*} A$, definida por

$$
m^{*} A=\inf _{A \subseteq \bigcup} \sum_{I_{n}} l\left(I_{n}\right)
$$

com a coleção $\left\{I_{n}\right\}_{n \in \mathbb{N}}$ variando entre as coleçöes de intervalos abertos que cobrem $A$, isto é, $A \subseteq \bigcup I_{n}$. A função de conjuntos $m^{*}$ é chamada Medida Exterior.

Proposição 1.5. A medida exterior de um intervalo é igual a seu comprimento.

Embora a medida exterior esteja definida para todos os subconjuntos de $\mathbb{R}$, ela não é $\sigma$-aditiva. Para os detalhes, veja Royden [6], Capítulo 3. No entanto, ela passa a satisfazer esta propriedade se restringirmos a família de conjuntos para a qual $m^{*}$ está definida. A definição seguinte foi proposta por Carathéodory.

Definição 1.6 (Conjunto Mensurável). Um conjunto $A \subseteq \mathbb{R}$ é dito mensurável (ou Lebesgue-mensurável) se, para todo subconjunto $B \subseteq \mathbb{R}$, tivermos

$$
m^{*} B=m^{*}(B \cap A)+m^{*}\left(B \cap A^{\mathrm{c}}\right)
$$


A família dos conjuntos mensuráveis é denotada por $\mathfrak{M}$. Na verdade, a condição $m^{*}(B) \leq m^{*}(B \cap A)+m^{*}\left(B \cap A^{\mathrm{c}}\right)$ está sempre satisfeita, bastando mostrar a desigualdade oposta. Notemos também que a definição 1.6 é simétrica com relação a $A$ e a seu conjunto complementar. Claramente, $\varnothing$ e $\mathbb{R}$ são mensuráveis.

Muitos dos próximos resultados serão apenas enunciados, por serem básicos e conhecidos.

Lema 1.7. Todo intervalo é mensurável.

Teorema 1.8. A coleção $\mathfrak{M}$ dos conjuntos Lebesgue-mensuráveis é uma $\sigma$-álgebra.

Na demonstração deste teorema, que pode ser encontrada em Royden [6], mostrase que $\mathfrak{M}$ é fechado quanto a uniões e intersecções finitas. Então, verifica-se o fechamento para uniões enumeráveis.

Corolário 1.9. Todo boreliano (veja seção 1.3) é mensurâvel. Em particular, todo conjunto aberto ou fechado é mensurável.

Como veremos na seção 1.3 , o conjunto $\mathcal{B}$, dos conjuntos borelianos, pode ser definido como a menor $\sigma$-álgebra que contém os abertos de $\mathbb{R}$. Não é difícil vermos que todo aberto é mensurável, pois é escrito como união enumerável de intervalos abertos, que são mensuráveis. Como $\mathcal{B} \subset \mathfrak{M}$, segue o resultado.

Se $A$ é um conjunto mensurável, definimos sua medida de Lebesgue, denotada por $m A$, como a medida exterior de $A$. Logo, a função $m$ é obtida pela restrição de $m^{*}$ à família $\mathfrak{M}$ dos conjuntos mensuráveis. Na verdade, $m$ é uma medida. Isto segue do fato (não imediato) de ser $m\left(\bigcup A_{i}\right)=\sum m A_{i}$, para toda coleção enumerável $\left\{A_{i}\right\}$ de conjuntos mensuráveis.

O lema a seguir dá condições para que a medida de Lebesgue $m$ seja completa.

Lema 1.10. Se $m^{*} A=0$, então A é mensurävel.

Demonstração: Seja $B \subseteq \mathbb{R}$. Como $B \cap A \subseteq A$, temos $m^{*}(B \cap A) \leq m^{*} A=0$. Também, $B \supseteq B \cap A^{\mathrm{c}}$. Logo, $m^{*} B \geq m^{*}\left(B \cap A^{\mathrm{c}}\right)=m^{*}\left(B \cap A^{\mathrm{c}}\right)+m^{*}(B \cap A)$, que implica $A$ é mensurável. 


\subsection{Medidas com Sinal}

Notemos que, se $\mu_{1}$ e $\mu_{2}$ são duas medidas positivas definidas em um espaço mensurável $(X, \mathcal{A})$, então $\mu_{3}$, definida por

$$
\mu_{3} E=c_{1} \mu_{1} E+c_{2} \mu_{2} E, \quad c_{1}, c_{2} \geq 0 .
$$

é uma nova medida positiva em $(X, \mathcal{A})$. O que pode ocorrer se tentarmos definir uma medida $\nu$ por $\nu E=\mu_{1} E-\mu_{2} E$ ? Primeiramente, $\nu$ pode assumir valores negativos. Isto nos diz que $\nu$ pode não ser uma medida no sentido da definição 1.2. Uma dificuldade mais grave vem do fato de $\nu$ não estar definida quando $\mu_{1} E=\mu_{2} E=\infty$. Por esta razão, alguma das medidas $\mu_{1}$ ou $\mu_{2}$ deve ser finita, se quisermos definir a função $\nu$ tomando valores em $\overline{\mathbb{R}}$. Tendo estas observações em mente, podemos definir o conceito de medida com sinal.

Definição 1.11 (Medida com Sinal). Uma medida com sinal $\mu$, definida em uma $\sigma$-álgebra $\mathcal{A}$, é uma função real estendida satisfazendo as condições

i. $\mu$ assume, no máximo, um dos valores $+\infty,-\infty$;

ii. $\mu(\varnothing)$ é igual a zero;

iii. Para toda família disjunta $\left\{A_{i}\right\}_{i \in \mathbb{N}}$ de conjuntos mensuráveis,

$$
\mu\left(\bigcup_{i=1}^{\infty} A_{i}\right)=\sum_{i=1}^{\infty} \mu\left(A_{i}\right)
$$

e, caso $\mu\left(\bigcup A_{i}\right)$ seja finita, a série no segundo membro converge absolutamente.

Como já dissemos, uma medida com sinal também será chamada por "medida". Usaremos o termo "medida com sinal" onde houver ambigüidade com o conceito da definição 1.2. Dizemos que um conjunto mensurável $A$ é positivo com respeito a uma medida $\mu$ se $\mu \tilde{A} \geq 0$ para todo subconjunto mensurável $\tilde{A} \subseteq A$. Todo subconjunto mensurável de um conjunto positivo é também positivo. A restrição de $\mu$ a um conjunto positivo é uma medida positiva. Similarmente, um conjunto $B$ é dito negativo se $\mu \tilde{B} \leq 0$ para $\tilde{B}$ mensurável, $\tilde{B} \subseteq B$. Um conjunto positivo e negativo é dito nulo. Note que um conjunto com medida $\mu$ positiva, negativa ou nula não é necessariamente positivo, negativo ou nulo, respectivamente. Podemos 
decompor um espaço de medida em termos destes conjuntos e, então, escrevermos $\mu$ como a diferença de duas medidas positivas. Este resultado é conhecido como Teorema da Decomposição de Hahn.

Teorema 1.12 (Decomposição de Hahn). Seja $\mu$ uma medida definida em um espaço mensurâvel $(X, \mathcal{A})$. Então existe um conjunto positivo $A$ e um conjunto negativo $B$ tais que $X=A \cup B$ e $A \cap B=\varnothing$. Esta decomposiçāo é única a menos de conjuntos nulos. O par $\{A, B\}$ é chamado uma decomposição de Hahn para $\mu$.

Este teorema e sua demonstração podem ser encontrados em Royden [6], como Teorema 11.21.

Se $\{A, B\}$ é uma decomposição de Hahn para uma medida $\mu$, podemos definir duas medidas positivas $\mu^{+}$e $\mu^{-}$, com $\mu=\mu^{+}-\mu^{-}$, definindo

$$
\begin{aligned}
\mu^{+} E & =\mu(E \cap A) \\
\mu^{-} E & =-\mu(E \cap B) .
\end{aligned}
$$

Estas medidas satisfazem $\mu^{-} A=0=\mu^{+} B$. Como $\mu$ não assume ambos os valores $+\infty,-\infty$, uma das medidas $\mu^{+}, \mu^{-}$deve ser finita. A medida positiva $|\mu|$ definida por

$$
|\mu| E=\mu^{+} E+\mu^{-} E
$$

é chamada a medida variação total de $\mu$. Um conjunto $E$ é positivo se $\mu^{-} E=0$, negativo se $\mu^{+} E=0$ e nulo se $|\mu| E=0$.

\subsection{Medidas Geradas por Funções}

Dada qualquer coleção $\mathfrak{A}$ de subconjuntos de $X$, existe uma menor $\sigma$-álgebra $\mathcal{A}$ que contém $\mathfrak{A}$, ou seja, se $\tilde{\mathcal{A}}$ é uma $\sigma$-álgebra que contém $\mathfrak{A}$, então $\mathcal{A} \subseteq \tilde{\mathcal{A}}$. $\grave{A}$ menor $\sigma$-álgebra que contém os conjuntos abertos de um conjunto $A \subseteq \mathbb{R}$, damos o nome de $\sigma$-Álgebra de Borel, denotada por $\mathcal{B}=\mathcal{B}(A)$, e chamamos seus elementos de borelianos. Uma medida $\mu$ definida em $\mathcal{B}$, finita para conjuntos limitados, é chamada uma medida de Borel. A cada medida de Borel positiva finita $\mu$, associamos uma função $F$ definida por

$$
F(x)=\mu(-\infty, x]
$$


A função $F$ assume valores reais e é crescente ${ }^{2}$. Temos

$$
\mu(a, b]=F(b)-F(a)
$$

Como $(a, b]$ é a interseç̧ão dos conjuntos $\left(a, b+\frac{1}{n}\right], n \in \mathbb{N}$, concluímos, pela Proposição 1.3, que

$$
\mu(a, b]=\lim _{n \rightarrow \infty} \mu\left(a, b+\frac{1}{n}\right]
$$

e, portanto, $F(b)=\lim _{n \rightarrow \infty} F\left(b+\frac{1}{n}\right)=F(b+)$, ou seja, $F$ é contínua à direita. Similarmente,

$$
\begin{aligned}
\mu\{b\} & =\lim _{n \rightarrow \infty}\left(b-\frac{1}{n}, b\right] \\
& =\lim _{n \rightarrow \infty} F(b)-F\left(b-\frac{1}{n}\right) \\
& =F(b)-F(b-) .
\end{aligned}
$$

Também, por $\varnothing=\bigcap_{n=1}^{\infty}(-\infty,-n]$, temos que

$$
\lim _{n \rightarrow-\infty} F(n)=\lim _{n \rightarrow-\infty} \mu(-\infty, n]=\mu(\varnothing)=0
$$

Como $F$ é crescente, $\lim _{x \rightarrow-\infty} F(x)=0$.

Temos um resultado sobre a recíproca destes fatos, mesmo para funções crescentes que não satisfaçam a todas as condições mostradas acima.

Proposição 1.13. Seja $F$ uma função cresčente, contínua d direita e definida em um intervalo $I \subseteq \mathbb{R}$. Então existe uma única medida de Borel positiva $d f$, dita gerada por $f$, tal que, para a e bem $I$, com $a<b$, temos

$$
d f(a, b]=F(b)-F(a)
$$

Também, se F for limitada, então df é finita.

Esta medida, cujo domínio é $\mathcal{B}$, é conhecida como medida de Borel-Stieltjes. Mais adiante, veremos que uma importante classe de funções (funções de variação limitada) é caracterizada pelas funções monótonas. Para estas funções, podemos definir uma medida no mesmo espírito do que foi feito no tópico sobre medidas com sinal.

\footnotetext{
${ }^{2}$ Neste texto, dizemos que uma função $f$ é crescente se $f(x) \leq f(y)$ sempre que $x \leq y$.
} 
Proposição 1.14. Seja $f=f^{+}-f^{-}$, com $f^{+}, f^{-}$funções crescentes definidas em um intervalo I e continuas à direita. Entāo existe uma única medida com sinal df definida em $\mathcal{B}(K)$, sendo $K \subseteq$ I qualquer intervalo onde alguma das $f^{+}, f^{--}$é limitada, tal que, para a e bem $K$, com $a<b$, temos

$$
d f(a, b]=f(b)-f(a)
$$

Teremos, no caso da proposição anterior, $d f=d f^{+}-d f^{-}$, sendo as medidas $d f^{+}, d f^{-}$geradas pelas funções $f^{+}, f^{--}$, respectivamente. A medida $d f$ independe da decomposição de $f$ como diferença de funções crescentes. A medida $d f$ é conhecida como medida de Borel-Stieltjes. Se $f$ é a função identidade em $\mathbb{R}$, ou seja, $d f(a, b]=$ $b-a$, que é o comprimento do intervalo $(a, b]$, df é a medida de Lebesgue restrita a B.

\section{Integração}

Nas próximas seções, utilizaremos largamente as integrais de Stieltjes, como nos tópicos sobre distribuições, a representação integral das EDM, etc. Novamente, este capítulo tem o intuito de recordar os resultados principais dessa teoria de integraçāo. As integrais de Lebesgue, de Riemann-Stieltjes e Lebesgue-Stieltjes serão o enfoque desta seção. Não apresentaremos as demonstrações de alguns resultados a seguir, as quais podem ser encontradas nos textos de Rudin [7] ou Royden [6]. Aqui, os conjuntos mensuráveis são caracterizados pela Definição 1.6.

\subsection{Integral de Riemann-Stieltjes}

As funções integráveis no sentido de Riemann-Stieltjes relativamente a uma função $\alpha$ possuem algumas boas propriedades que serão aproveitadas em suas integrais de Lebesgue-Stieltjes, através do Teorema 1.27, como por exemplo a fórmula de integração por partes e a fórmula da mudança da função $\alpha$ de integração.

Seja $f$ uma função limitada e $\alpha$ uma função crescente, ambas definidas em $[a, b]$. Para cada partição $\pi: a=t_{0}<t_{1}<\cdots<t_{n}=b$, consideramos os valores

$$
U(\pi, f, \alpha)=\sum_{i=1}^{n} M_{i}\left[\alpha\left(t_{i}\right)-\alpha\left(t_{i-1}\right)\right], \quad L(\pi, f, \alpha)=\sum_{i=1}^{n} m_{i}\left[\alpha\left(t_{i}\right)-\alpha\left(t_{i-1}\right)\right],
$$


sendo $M_{i}=\sup _{t_{i} \leq t \leq t_{i-1}} f(x), m_{i}=\inf _{t_{i} \leq t \leq t_{i-1}} f(x)$. Assim, os valores

$$
\begin{aligned}
& \overline{\mathrm{R}} \int_{a}^{b} f d \alpha=\inf _{\pi} U(\pi, f, \alpha) \\
& \underline{\mathrm{R}} \int_{a}^{b} f d \alpha=\sup _{\pi} L(\pi, f, \alpha)
\end{aligned}
$$

são as somas superior e inferior de Riemann, respectivamente, de $f$ relativamente a $\alpha$. Se os valores em (1.1) e (1.2) são iguais, designamos este valor comum por

$$
\mathrm{R} \int_{a}^{b} f d \alpha
$$

ou, às vezes, por $\mathrm{R} \int_{a}^{b} f(x) d \alpha(x)$, e dizemos que $f$ é Riemann-integrável relativamente a $\alpha$, escrevendo $f \in \mathfrak{R}(\alpha)$. Esta é a integral de Riemann-Stieltjes de $f$ com relação $a \alpha$ em $[a, b]$. No caso em que $\alpha(x)=x$, a integral é conhecida apenas por integral de Riemann de $f$. Neste caso, escrevemos $f \in \mathfrak{R}$ e a integral como $R \int_{a}^{b} f(x) d x$ ou, simplesmente, $\mathrm{R} \int_{a}^{b} f$.

Teorema 1.15. $f \in \mathfrak{R}(\alpha)$ em $[a, b]$ se e somente se para cada $\varepsilon>0$, existe uma partiçāo $\pi$ de $[a, b]$ tal que $U(\pi, f, \alpha)-L(\pi, f, \alpha)<\varepsilon$.

Para uma partição $\pi: a=t_{0}<t_{1}<\cdots<t_{n}=b$, definimos sua amplitude como $\lambda(\pi)=\max _{i}\left(t_{i}-\dot{t}_{i-1}\right)$. Dada ùma função $\alpha$, para uma partição $\pi$, escrevemos

$$
\Delta \alpha_{i}=\left[\alpha\left(t_{i}\right)-\alpha\left(t_{i-1}\right)\right]
$$

Teorema 1.16. Se f é contínua em $[a, b]$, então f é Riemann-integrável com relação a $\alpha$.

Demonstração: Dado $\varepsilon>0$, seja $\eta>0$ tal que $[\alpha(b)-\alpha(a)] \eta<\varepsilon$. Como $f$ é contínua em $[a, b]$, e portanto uniformemente contínua, existe $\delta>0$ tal que

$$
|f(x)-f(t)|<\eta
$$

sempre que $|x-t|<\delta$. Escolhamos $\pi$ tal que $\lambda(\pi)<\delta$. Decorre de (1.3) que $M_{i}-m_{i} \leq \eta, i=1, \cdots, n$. Portanto

$$
U(\pi, f, \alpha)-\dot{L}(\pi, f, \alpha)=\sum_{i=1}^{n}\left(M_{i}-m_{i}\right) \Delta \alpha_{i} \leq \sum_{i=1}^{n} \eta \Delta \alpha_{i}=\eta[\alpha(b)-\alpha(a)]<\varepsilon .
$$

Pelo Teorema 1.15, $f$ é Riemann-integrável com relação a $\alpha$. 
Teorema 1.17. Se $f$ é monótona em $[a, b]$ e se $\alpha$ é contínua (e crescente), então $f$ é Riemann-integrável com relação a $\alpha$.

Demonstração: Dado $\varepsilon>0$, qualquer que seja $m$, um inteiro positivo, existe uma partição $\pi$ tal que

$$
\Delta \alpha_{i} \leq \frac{\alpha(b)-\alpha(a)}{m}, \quad i=1, \ldots, n
$$

Isto é possível pois $\alpha$ é contínua. Suponhamos, sem perda de generalidade, que $f$ é monótona crescente. Nestas condições, $M_{i}=f\left(t_{i}\right)$ e $m_{i}=f\left(t_{i-1}\right)$. Entāo

$$
\begin{aligned}
U(\pi, f, \alpha)-L(\pi, f, \alpha) & =\sum_{i=1}^{n}\left[f\left(t_{i}\right)-f\left(t_{i-1}\right)\right] \Delta \alpha_{i} \\
& \leq[f(b)-f(a)] \cdot \frac{\alpha(b)-\alpha(a)}{m}<\varepsilon
\end{aligned}
$$

desde que $m$ seja suficientemente grande. Pelo Teorema $1.15, f \in \mathfrak{R}(\alpha)$.

Seja $\pi$ uma partição e $u_{1}, \ldots, u_{n}$ tais que $t_{i-1} \leq u_{i} \leq t_{i}$. Definimos

$$
S(\pi, f, \alpha)=\sum_{i=1}^{n} f\left(u_{i}\right) \Delta \alpha_{i} .
$$

como a soma de $f$ com relação a $\alpha$ por $\pi$, que depende também da escolha dos $u_{i}$.

O teorema seguinte ilustra quando a integral de Riemann-Stieltjes é limite das somas de funções por partiçōes cada vez mais finas.

Teorema 1.18. i. Se $\lim _{\lambda(\pi) \rightarrow 0} S(\pi, f, \alpha)$ existe, independente da escolha dos pontos $u_{1}, \ldots, u_{n}$, então $f \in \mathfrak{R}(\alpha) e$

$$
\lim _{\lambda(\pi) \rightarrow 0} S(\pi, f, \alpha)=\mathrm{R} \int_{a}^{b} f d \alpha .
$$

ii. Se (i) $f$ é contínua em $[a, b]$ ou se (ii) f é monótona e $\alpha$ é contínua e crescente em $[a, b]$, então (1.4) se verifica.

O próximo teorema ilustra quando as integrais de Riemann-Stieltjes se reduzem a integrais de Riemann. Ele também é muito útil nas substituições das funções peso, na integração de Riemann-Stieltjes. 
Teorema 1.19. Se $f \in \mathfrak{R}$ e $\alpha^{\prime} \in \mathfrak{R}$ em $[a, b]$, então $f \in \mathfrak{R}(\alpha)$ e

$$
\mathrm{R} \int_{a}^{b} f d \alpha=\mathrm{R} \int_{a}^{b} f(x) \alpha^{\prime}(x) d x
$$

Demonstração: Seja $\varepsilon>0$. Consideremos $M$ tal que $|f|<M$. Como $f \alpha^{\prime} \in \mathfrak{R} \mathrm{e}$ $\alpha^{\prime} \in \mathfrak{R}$, o Teorema 1.18 mostra que existem $\delta_{1}, \delta_{2}>0$ tais que

$$
\left|\sum f\left(u_{i}\right) \alpha^{\prime}\left(u_{i}\right)\left[t_{i}-t_{i-1}\right]-\mathrm{R} \int f \alpha^{\prime}\right|<\varepsilon
$$

se $\lambda(\pi)<\delta_{1}$ e $t_{i-1} \leq u_{i} \leq t_{i} ;$ e

$$
\left|\sum \alpha^{\prime}\left(u_{i}\right)\left[t_{i}-t_{i-1}\right]-\mathrm{R} \int \alpha^{\prime}\right|<\varepsilon
$$

se $\lambda(\pi)<\delta_{2}$ e $t_{i-1} \leq u_{i} \leq t_{i}$. Variando $u_{i}$ nesta última desigualdade, vemos que

$$
\sum\left|\alpha^{\prime}\left(u_{i}\right)-\alpha^{\prime}\left(v_{i}\right)\right|\left[t_{i}-t_{i-1}\right]<2 \varepsilon
$$

se $\lambda(\pi)<\delta_{2}$ e $t_{i-1} \leq u_{i}, v_{i} \leq t_{i}$

Agora, considere $\pi$ tal que $\lambda(\pi)<\delta=\min \left\{\delta_{1}, \delta_{2}\right\}$ e seja $u_{i} \in\left[t_{i-1}, t_{i}\right]$. Existe $v_{i} \in\left[t_{i-1}, t_{i}\right]$ tais que $\left[\alpha\left(t_{i}\right)-\alpha\left(t_{i-1}\right)\right]=\alpha^{\prime}\left(v_{i}\right)\left[t_{i}-t_{i-1}\right]$, pelo Teorema do Valor Médio. Então

$$
\sum f\left(u_{i}\right) \Delta \alpha_{i}=\sum f\left(u_{i}\right) \alpha^{\prime}\left(u_{i}\right)\left[t_{i}-t_{i-1}\right]+\sum f\left(u_{i}\right)\left[\alpha^{\prime}\left(v_{i}\right)-\alpha^{\prime}\left(u_{i}\right)\right]\left[t_{i}-t_{i-1}\right] .
$$

Por (1.5) e (1.6), a diferença entre o primeiro membro de (1.7) e $\mathrm{R} \int f \alpha^{\prime}$ é menor que $(2 M+1) \varepsilon$. Isto significa

$$
\lim _{\lambda(\pi) \rightarrow 0} S(\pi, f, \alpha)=\mathrm{R} \int_{a}^{b} f(x) \alpha^{\prime}(x) d x
$$

o que completa a demonstração, pelo Teorema 1.18.

Teorema 1.20 (Integração por Partes). Se $f$ e $\alpha$ são funções crescentes em $[a, b]$ e $f \hat{e}$ contínua em $[a, b]$, então

$$
\mathrm{R} \int_{a}^{b} f d \alpha=f(b) \alpha(b)-f(a) \alpha(a)-\mathrm{R} \int_{a}^{b} \alpha d f .
$$


Demonstração: Seja $\pi: a=t_{0}<\cdots<t_{n}=b$ uma partição de $[a, b]$. Tomando $u_{i}=t_{i-1}$, por adição por partes ${ }^{3}$, temos

$$
\begin{aligned}
S(\pi, f, \alpha) & =\sum_{i=1}^{n} f\left(t_{i-1}\right)\left[\alpha\left(t_{i}\right)-\alpha\left(t_{i-1}\right)\right] \\
& =f(b) \alpha(b)-f(a) \alpha(a)-\sum_{i=1}^{n} \alpha\left(t_{i}\right)\left[f\left(t_{i}\right)-f\left(t_{i-1}\right)\right] \\
& =f(b) \alpha(b)-f(a) \alpha(a)-S(\pi, \alpha, f) .
\end{aligned}
$$

Quando $\lambda(\pi) \rightarrow 0$, o Teorema 1.18 nos dá $S(\pi, f, \alpha) \rightarrow \mathrm{R} \int_{a}^{b} f d \alpha$ e $S(\pi, \alpha, f) \rightarrow$ $\mathrm{R} \int_{a}^{b} \alpha d f$.

\subsection{Integral de Lebesgue-Stieltjes}

Proposição 1.21. Seja $F$ uma função tomando a em $\overline{\mathbb{R}}$ cujo domínio é mensurável. Seja $\alpha \in \overline{\mathbb{R}}$. Então as seguintes afirmaçōes sāo equivalentes:

i. O conjunto $\{x: F(x)>\alpha\}$ é mensurâvel.

ii. O conjunto $\{x: F(x) \geq \alpha\}$ é mensurâvel.

iii. O conjunto $\{x: F(x)<\alpha\}$ é mensurável.

iv. O conjunto $\{x: F(x) \leq \alpha\}$ é mensurável.

Estas afirmações implicam

v. O conjunto $\{x: F(x)=\alpha\}$ é mensurável.

Definição 1.22 (Função Mensurável). Uma função é dita mensurável se seu domínio é mensurável e se satisfaz a alguma das primeiras quatro afirmaçōes da proposição 1.21 para todo $\alpha \in \overline{\mathbb{R}}$.

Lema 1.23. Se cé uma constante e $f, g$ são funções mensuráveis, então $f+c, c f, f+g$ e fg são também funções mensuráveis. Além disso, se $\left(f_{n}\right)$ é uma seqüência de funções mensuráveis, entäo $\sup f_{n}, \inf f_{n}, \underline{\lim } f_{n} e \varlimsup \overline{\lim } f_{n}$ sāo mensuráveis.

${ }^{3} \mathrm{Se}\left(a_{n}\right),\left(b_{n}\right), n=0,1, \cdots$, são seqüências de números reais, a identidade $\sum_{i=1}^{n} a_{i}\left(b_{i}-b_{i-1}\right)=$ $a_{n} b_{n}-a_{0} b_{0}-\sum_{i=1}^{n} b_{i-1}\left(a_{i}-a_{i-1}\right)$ é conhecida como Adição por Partes. 
Por uma função simples entendemos uma combinação linear finita

$$
\varphi(x)=\sum_{i=1}^{n} c_{i} \chi_{E_{i}}(x)
$$

de funções características de conjuntos mensuráveis $E_{i}$, ou seja, $\chi_{E_{i}}(x)=1$ se $x \in E_{i}$ e $\chi_{E_{i}}(x)=0$, caso contrário. Para uma função simples $\varphi$, dada como em (1.8), definimos sua integral, com respeito a uma medida $\mu$, sobre um conjunto mensurável $E$ por

$$
\int_{E} \varphi d \mu=\sum_{i=1}^{n} c_{i} \mu\left(E_{i} \cap E\right) .
$$

Esta definição independe da representação de $\varphi$ como função simples. Se $f$ é uma função real mensurável limitada em $E$, um conjunto mensurável, podemos definir a integral de $f$ com respeito à medida $\mu$ por analogia à integral de Riemann, considerando os números

$$
\sup _{\varphi \leq f} \int_{E} \varphi d \mu \quad \text { e } \inf _{\psi \geq f} \int_{E} \psi d \mu
$$

e perguntando quando estes valores são iguais. A proposição seguinte nos dá esta resposta.

Proposição 1.24. Seja $f$ uma função definida e limitada em um conjunto mensurável $E$ $\operatorname{com} \mu E$ finita. Então

$$
\sup _{f \geq \varphi} \int_{E} \varphi(x) d x=\inf _{f \leq \psi} \int_{E} \psi(x) d x
$$

com $\varphi$ e $\psi$ funções simples, se e somente se f é mensurável.

Demonstração: Suponhamos que $f$ seja mensurável. Seja $M$ tal que $|f| \leq M$ e $n$ um número natural. Então os conjuntos

$$
E_{k}=\left\{x \in E: \frac{(k-1) M}{n}<f(x) \leq \frac{k M}{n}\right\},-n \leq k \leq n,
$$

são mensuráveis, disjuntos e têm união $E$. Logo, $\sum_{k=-n}^{n} m E_{k}=m E$. As funções simples definidas como

$$
\begin{aligned}
\varphi_{n}(x) & =\frac{M}{n} \sum_{k=-n}^{n}(k-1) \chi_{E_{k}}(x) \\
\psi_{n}(x) & =\frac{M}{n} \sum_{k=-n}^{n} k \chi_{E_{k}}(x)
\end{aligned}
$$


satisfazem $\varphi_{n}(x) \leq f(x) \leq \psi_{n}(x)$. Das desigualdades

$$
\begin{aligned}
& \sup _{\varphi \leq f} \int_{E} \varphi d \mu \geq \int_{E} \varphi_{n} d \mu=\frac{M}{n} \sum_{k=-n}^{n}(k-1) \mu E_{k} \\
& \inf _{\psi \leq f} \int_{E} \psi d \mu \leq \int_{E} \psi_{n} d \mu=\frac{M}{n} \sum_{k=-n}^{n} k \mu E_{k},
\end{aligned}
$$

tiramos

$$
0 \leq \inf _{\psi \leq f} \int_{E} \psi d \mu-\sup _{\varphi \leq f} \int_{E} \varphi d \mu \leq \frac{M}{n} \sum_{k=-n}^{n} \mu E_{k}=\frac{M}{n} \mu E .
$$

Por $n$ ser arbitrário, segue o resultado. A recíproca não será mostrada neste texto. No entanto, sua demonstração pode ser encontrada em Royden [6, Th. 4.3].

Definição 1.25 (Integral de Lebesgue-Stieltjes). Seja $f$ uma função mensurável definida em um espaço de medida $(X, \mathcal{A}, \mu)$ limitada em $E$, um conjunto mensurável. Definimos a integral de Lebesgue-Stieltjes de $f$ em $E$, com relação à medida $\mu$ pelo valor comum aos valores em (1.9), ou seja,

$$
\int_{E} f d \mu=\sup _{\varphi \leq f} \int_{E} \varphi d \mu=\inf _{\psi \geq f} \int_{E} \psi d \mu,
$$

$\operatorname{com} \varphi$ e $\psi$ funções simples.

Definição 1.26 (Função Integrável). Uma função $\mu$-mensurável $f$ é dita $\mu$-integrável em A se

$$
\int_{A} f d \mu<\infty .
$$

Seja $\alpha$ uma função escrita como diferença de duas funções crescentes (ver Proposição 1.14), e $d \alpha$ a medida gerada por $\alpha$. Algumas vezes, indicamos a variável de integração $t$ escrevendo a integral $\int_{E} f d \alpha$ como

$$
\int_{E} f(t) d \alpha(t)
$$

Se $\alpha(t)=t, d \alpha$ é a medida de Lebesgue. A integral sobre esta medida é conhecida como integral de Lebesgue, e escrevemos

$$
\int f(t) d t
$$


ou simplesmente $\int f$. Se $E$ é deixado como variável, $\int_{E} f d \alpha$ é chamada a integral indefinida de $f$ com respeito a $\alpha$.

É usual entendermos

$$
\int_{a}^{b} f d \alpha=\int_{[a, b]} f d \alpha .
$$

A integral de Lebesgue possui a útil propriedade de, para $a<b<c$,

$$
\int_{a}^{b} f d t+\int_{b}^{c} f d t=\int_{a}^{c} f d t .
$$

Podemos fazer com que as integrais de Lebesgue-Stieltjes satisfaçam essa propriedade, definindo

$$
\int_{a}^{b} f d \alpha=\int_{(a, b]} f d \alpha .
$$

Se $\alpha$ é contínua em $a$, então $d \alpha\{a\}=0$, e as definições em (1.10) e (1.11) são equivalentes. Por isso, a integral $\int_{a}^{b} f d \alpha$ será entendida como em (1.11) nos próximos capítulos.

Como $|\mu|(I)=V(f, I)$ (veja a Seção 4) quando $I$ é um subintervalo de $(a, b]$, a integral $\int_{(a, b]} f d|\mu|$ é denotada por

$$
\int_{a}^{b} f d v_{\alpha}
$$

Além disso, vale a relação

$$
\left|\int_{a}^{b} f d \alpha\right| \leq \int_{a}^{b}|f(t)| d v_{\alpha}(t) .
$$

O próximo teorema nos diz como a integral de Lebesgue-Stieltjes pode ser vista como uma generalização da integral de Riemann-Stieltjes.

Teorema 1.27. Seja $f$ uma função limitada em $[a, b]$. Se $\alpha$ é uma função contínua à direita e de variação limitada, e f é Riemann-integrável com relação a $\alpha$, então $f$ é mensurável e

$$
\mathrm{R} \int_{a}^{b} f d \alpha=\int_{a}^{b} f d \alpha
$$


Demonstração: Seja $\mu$ a medida gerada por $\alpha$. Como na Seção 2.1, sejam $\pi: a=$ $t_{0}<\cdots<t_{n}=b$ uma partição de $[a, b], M_{i}=\sup _{t_{i-1} \leq t \leq t_{i}} f(t)$ e $m_{i}=\sup _{t_{i-1} \leq t \leq t_{i}} f(t)$. Definamos as funções simples

$$
\varphi=\sum_{i=1}^{n} m_{i} \chi_{\left(t_{i-1}, t_{i}\right]}, \quad \psi=\sum_{i=1}^{n} M_{i} \chi_{\left(t_{i-1}, t_{i}\right)} .
$$

Como $\alpha$ é contínua à direita, $\mu\left(t_{i-1}, t_{i}\right]=\alpha\left(t_{i}\right)-\alpha\left(t_{i-1}\right)$. Nestas condições,

$$
L(\pi, f, \alpha)=\int_{a}^{b} \varphi d \alpha, \quad U(\pi, f, \alpha)=\int_{a}^{b} \psi d \alpha .
$$

Variando $\pi$ entre todas as partições de $[a, b]$, tiramos

$$
\underline{\mathrm{R}} \int_{b}^{a} f d \alpha \leq \sup _{\varphi \leq f} \int_{a}^{b} \varphi d \alpha \leq \inf _{\psi \leq f} \int_{a}^{b} \psi d \alpha \leq \overline{\mathrm{R}} \int_{b}^{a} f d \alpha .
$$

Como $f$ é Riemann-integrável com relação a $\alpha$, as desigualdades são todas igualdades. Segue da recíproca do Teorema 1.24 que $f$ é mensurável.

O resultado seguinte versa sobre quando podemos comutar o símbolo da integral de Lebesgue-Stieltjes com o limite.

Teorema 1.28 (Convergência Dominada de Lebesgue). Seja $(X, \mathcal{A}, \mu)$ um espaço de medida. Se gé $\mu$-integrável em $X e\left(f_{n}\right)$ é uma seqüência de funções $\mu$-mensuráveis tal que $f_{n}(x) \rightarrow f(x) e\left|f_{n}(x)\right| \leq g(x)$ para quase todo $x \in X$, ou seja, a menos de um conjunto do qual medida $\mu$ é nula, então

$$
\int_{X} f d \mu=\lim \int_{X} f_{n} d \mu
$$

A demonstração deste fato pode ser encontrada em Royden [6, Th. 11.16].

\section{Integral de Funções a Valores em $\mathbb{R}^{n}$}

Consideremos uma função $f: E \subseteq \mathbb{R} \rightarrow \mathbb{R}^{n}$. Chamemos suas funções coordenadas por $f^{i}, 1 \leq i \leq n$. Se $\alpha$ é contínua à direita e é diferença de funções crescentes, para A mensurável, $A \subseteq E$, entendemos o símbolo

$$
\int_{A} f d \alpha
$$


como o valor $\mathrm{x}$ em $\mathbb{R}^{n}$ cujas coordenadas $\mathrm{x}^{i}$ são dadas por

$$
\mathbf{x}^{i}=\int_{A} f^{i}(t) d \alpha(t), \quad 1 \leq i \leq n
$$

Com isto, vale a relação

$$
\left|\int_{A} f(t) d \alpha(t)\right| \leq \int_{A}|f(t)| d v_{\alpha}(t) .
$$

\section{Diferenciabilidade}

No intuito de falarmos sobre derivada de uma função, definamos quatro quantidades, chamadas de Derivadas de Dini.

Definição 1.29 (Derivadas de Dini). Seja $f: I \subseteq \mathbb{R} \rightarrow \mathbb{R}$. As quantidades

$$
\begin{aligned}
& D^{+} f(x)=\varlimsup_{h \rightarrow 0+} \frac{f(x+h)-f(x)}{h} \\
& D_{+} f(x)=\varliminf_{h \rightarrow 0+} \frac{f(x+h)-f(x)}{h} \\
& D^{-} f(x)=\varlimsup_{h \rightarrow 0-} \frac{f(x+h)-f(x)}{h} \\
& D_{-} f(x)=\varliminf_{h \rightarrow 0-} \frac{f(x+h)-f(x)}{h}
\end{aligned}
$$

sāo chamadas de Derivadas de Dini. As derivadas de Dini à direita $\left(D^{+}\right.$e $\left.D_{+}\right)$e à esquerda ( $D^{-}$e $\left.D_{-}\right)$existem somente se $x$ é ponto de acumulação em I à direita e à esquerda, respectivamente.

Claramente, temos $D^{+} f(x) \geq D_{+} f(x)$ e $D^{-} f(x) \geq D_{-} f(x)$.

Definição 1.30 (Diferenciabilidade). Dizemos que $f$ é diferenciável em $x$ se

$$
D^{+} f(x)=D^{-} f(x)=D_{+} f(x)=D_{-} f(x) \neq \pm \infty
$$

$e$ definimos $f^{\prime}(x)$ pelo valor comum das derivadas de Dini. Se tivermos $D^{+} f(x)=$ $D_{+} f(x) \neq \pm \infty$, dizemos que $f$ é diferenciável à direita em $x$. Similarmente, dizemos que $f \hat{e}$ diferenciável à esquerda em $x$ quando $D^{-} f(x)=D_{-} f(x) \neq \pm \infty$.

Para uma função crescente $f, D^{+} f(x)=D_{+} f(x)$ e $D^{-} f(x)=D_{-} f(x)$ sempre que estes valores existem e temos um importante resultado sobre diferenciabilidade dessas funções. 
Teorema 1.31. Seja $f$ uma função real crescente no intervalo $[a, b]$. Então $f$ é diferenciável em quase toda parte. A derivada $f^{\prime}$ é mensurável e

$$
\int_{a}^{b} f^{\prime}(x) d x \leq f(b)-f(a)
$$

Por ser extensa, omitiremos a prova do Teorema 1.31. No entanto, este teorema e sua demonstração podem ser encontrados em Royden [6, Th. 5.2].

\section{Funções de Variação limitada}

Seja $J=\left[t_{0}, \infty\right)$, e $f$ uma função definida em $J$ com valores em $\mathbb{R}^{n}$. A variação total de $f$ em $[a, b] \subset J$ é definida como

$$
V(f,[a, b])=\sup \left\{\sum_{i=1}^{n}\left|f\left(t_{i}\right)-f\left(t_{i-1}\right)\right|\right\},
$$

tomando o supremo sobre todas as possíveis partições $\pi: a=t_{0}<t_{1}<\cdots<t_{n}=b$ do intervalo $[a, b]$. Se $a<x<b$, vale a relação $V(f,[a, b])=V(f,[a, x])+V(f,[x, b])$. Dado $a \geq t_{0}$, dizemos que $f$ é de variação limitada em $[a, \infty)$ se $V(f,[a, t])<\infty$ para todo $t \in[a, \infty)$; veja Pandit-Deo [5] por exemplo. Se $f$ é função de variação limitada em um intervalo $I$ com extremo inferior $a$, então a função variação total de $f$, definida por

$$
v_{f}(t)=V(f,[a, t]), \quad t \in I
$$

é também de variação limitada e, além disso, é crescente. O espaço de todas as funções de variação limitada em $J$ tomando valores em $\mathbb{R}^{n}$ é denotado por $B V(J)=$ $B V\left(J, \mathbb{R}^{n}\right)$. Consideraremos sempre a norma de $f \in B V(J)$ definida por $\|f\|^{\star}=$ $V(f, J)+\left|f\left(t_{0}+\right)\right|$. Com esta norma, $B V(J)$ é espaço de Banach.

Consideremos, agora, funções a valores em $\mathbb{R}$. Funções de variação limitada e funções crescentes estão ligadas pelas duas proposições seguintes:

Proposição 1.32. Seja $f$ uma funçāo real crescente definida em um intervalo $[a, b]$. Então $f$ é de variação limitada em $[a, b]$.

Demonstração: Seja $\pi: a=t_{0}<\cdots<t_{n}=b$ uma partição de $[a, b]$. Como $f$ é crescente, temos $f\left(t_{i}\right)-f\left(t_{i-1}\right) \geq 0$. Portanto

$$
\sum_{i=1}^{n}\left|f\left(t_{i}\right)-f\left(t_{i-1}\right)\right|=\sum_{i=1}^{n} f\left(t_{i}\right)-f\left(t_{i-1}\right)=f(b)-f(a) .
$$


Como este valor é independente de $\pi$, segue que $f$ é de variação limitada. É fácil ver que $v_{f}(x)=f(x)-f(a)$.

Proposição 1.33. Seja $f$ uma função real de variação limitada definida em $[a, b]$. Então existem funções crescentes $f^{+}, f^{-}$tais que

$$
\begin{aligned}
f(x) & =f^{+}(x)-f^{-}(x)+f(a), \\
v_{f}(x) & =f^{+}(x)+f^{-}(x),
\end{aligned}
$$

para todo $x$ em $[a, b]$.

Demonstração: Definamos $f^{+}$e $f^{-}$por

$$
2 f^{+}=v_{f}+f-f(a), \quad 2 f^{-}=v_{f}-f+f(a) .
$$

É claro que $f^{+}(a)=f^{-}(a)=0$ e que (1.12) e (1.13) se verificam. Se $a \leq x \leq y \leq b$, temos

$$
\begin{aligned}
& 2 f^{+}(y)-2 f^{+}(x)=V(f,[x, y])+[f(y)-f(x)] \\
& 2 f^{-}(y)-2 f^{-}(x)=V(f,[x, y])-[f(y)-f(x)] .
\end{aligned}
$$

Como $|f(y)-f(x)| \leq V(f,[x, y])$, teremos $f^{+}$e $f^{-}$crescentes.

As funçōes $f^{+}$e $f^{-}$são chamadas função variação positiva e função variação negativa da função $f$, respectivamente. A equação (1.12) mostra que toda função de variação limitada pode ser escrita como diferença de funções crescentes, a saber $\left[f^{+}+f(a)\right]$ e $f^{-}$(por vezes, é conveniente escrevermos a decomposição anterior simplesmente por $f=f^{+}-f^{-}$, renomeando a função $f^{+}$). Isto faz com que as funções de variação limitada herdem algumas boas propriedades das funções crescentes. Uma delas está enunciada no corolário seguinte.

Corolário 1.34. Seja $f$ uma função real de variação limitada no intervalo $[a, b]$. Então $f$ é diferenciável em quase toda parte de $[a, b]$.

Podemos estender de maneira natural a integral de Riemann-Stieltjes de forma que as funções peso possam ser de variação limitada. Basta definirmos a integral $\mathrm{R} \int_{a}^{b} f d \alpha$ por

$$
\mathrm{R} \int_{a}^{b} f d \alpha=\mathrm{R} \int_{a}^{b} f d \alpha_{1}-\mathrm{R} \int_{a}^{b} f d \alpha_{2}
$$


sendo $\alpha=\alpha_{1}-\alpha_{2}$, com $\alpha_{1}, \alpha_{2}$ crescentes. Esta definição independe da representação de $\alpha$ como diferença de funções crescentes. Muitas vezes, não são criadas complicações quando transportamos alguns resultados de funções monótonas para funções de variação limitada.

Corolário 1.35 (do Teorema 1.20). Se $f$ e $\alpha$ são funções de variação limitada em $[a, b]$ e f é contínua em $[a, b]$, então

$$
\int_{a}^{b} f d \alpha=f(b) \alpha(b)-f(a) \alpha(a)-\int_{a}^{b} \alpha d f
$$

\subsection{Princípio da Seleção de Helly}

No caso do espaço das funções contínuas, conjuntos compactos são caracterizados pelo conhecido Teorema de Arzelá-Ascoli. Será mostrado, nesta seção, um resultado semelhante, porém mais fraco (no sentido de que a convergência é pontual), para o espaço das funções de variação limitada. Esse resultado terá sua aplicação no teorema de existência local de soluções de uma EDM (Teorema 2.4). Comecemos mostrando alguns resultados auxiliares.

O seguinte lema será usado na demonstração do Teorema 1.37.

Lema 1.36. Seja $\left(f_{n}\right)$ uma seqüência de funções reais definidas em um conjunto enumerável $E$ tal que, para cada $x$ em $E$, o conjunto $\left\{f_{n}(x): n \in \mathbb{N}\right\}$ seja limitado. Então existe uma subseqüência $\left(f_{n_{k}}\right)$ que converge pontualmente em $E$.

Demonstração: Seja $E=\left\{x_{i}\right\}$. Como $\left\{f_{n}\left(x_{1}\right): n \in \mathbb{N}\right\}$ é limitado, existe uma subseqüência $\left(f_{1 n}\right)$ de $\left(f_{n}\right)$ tal que $\left(f_{1 n}\left(x_{1}\right)\right)$ é convergente. Podemos extrair uma subseqüência $\left(f_{2 n}\right)$ de $\left(f_{1 n}\right)$ tal que $\left(f_{2 n}\left(x_{2}\right)\right)$ convirja. Continuando este processo, obtemos uma cadeia de subseqüências tal que $\left(f_{j n}\right)$ é convergente em $x_{j}$. Consideremos a seqüência "diagonal" $\left(f_{n n}\right)$. Temos que $\left(f_{n n}\right)_{n=j}^{\infty}$ é subseqüência de $\left(f_{j n}\right)$, e portanto $\left(f_{n n}\left(x_{j}\right)\right)$ é convergente.

Teorema 1.37. Seja $\left(\phi_{n}\right)$ uma seqüência limitada de funções crescentes definidas em $[a, b]$. Então existe uma subseqüência $\left(\phi_{n_{k}}\right)$ de $\left(\phi_{n}\right)$ que converge pontualmente para uma função $\phi$ crescente. 
Demonstração: Seja $E$ um conjunto enumerável denso em $[a, b]$, contendo os pontos extremos $a, b$. Pelo lema anterior, existe uma subseqüência $\left(\phi_{n_{k}}\right)$ convergente em $E$ para uma função crescente $\phi(x)$, definida inicialmente apenas em $E$. Para $x$ fora de $E$, podemos calcular os limites laterais

$$
\phi(x+)=\lim _{\substack{y \rightarrow x+\\ y \in E}} \phi(y), \quad \phi(x-)=\lim _{\substack{y \rightarrow x-\\ y \in E}} \phi(y) .
$$

Afirmamos que

$$
\begin{aligned}
& \varlimsup \\
& \varlimsup \\
& \underline{\lim } \phi_{n_{k}}(x) \leq \phi(x+), \\
& n_{n_{k}}(x) \geq \phi(x-) .
\end{aligned}
$$

De fato, se $\left(\varphi_{n}(x)\right)$ é uma subseqüência convergente de $\left(\phi_{n_{k}}(x)\right)$ e $y<x$, com $y \in E$, então

$$
\phi(y)=\lim \phi_{n_{k}}(y)=\lim \varphi_{n}(y) \leq \lim \varphi_{n}(x) .
$$

Fazendo $y$ tender a $x$ pela esquerda, teremos $\phi(x-) \leq \varliminf \phi(x)$. Mostra-se a outra identidade de maneira análoga.

Agora, se $x \notin E$ satisfaz $\phi(x-)=\phi(x+)$, a desigualdade

$$
\phi(x-) \leq \underline{\lim } \phi_{n_{k}}(x) \leq \varlimsup \overline{\lim } \phi_{n_{k}}(x) \leq \phi(x+)
$$

implica

$$
\underline{\varliminf} \phi_{n_{k}}(x)=\varlimsup \phi_{n_{k}}(x)=\lim \phi_{n_{k}}(x)
$$

e, portanto, $\left(\phi_{n_{k}}(x)\right)$ é convergente para um valor $\phi(x)$.

Seja $X_{N}=\left\{x \notin E:[\phi(x+)-\phi(x-)] \geq \frac{1}{N}\right\}$. A desigualdade

$$
\sum_{x \in X_{N}}[\phi(x+)-\phi(x-)] \leq \phi(b)-\phi(a)
$$

implica que o conjunto $X_{N}$ é finito. Se $x \notin E$ é tal que $\phi(x+) \neq \phi(x-)$, então $x$ está em $X_{N}$ para algum $N$. Logo, o conjunto dos pontos $x \notin E$ tais que $\phi(x+) \neq \phi(x-)$ é enumerável. Usando novamente $\circ$ Lema 1.36, podemos extrair uma subseqüência de $\left(\phi_{n_{k}}\right)$, que novamente chamaremos $\left(\phi_{n_{k}}\right)$, tal que $\left(\phi_{n_{k}}(x)\right)$ é convergente para todo $x$ pertencente $\mathrm{a}[a, b]$.

Seja $\mathfrak{F}=\left\{\phi_{\lambda}\right\}_{\lambda \in \Lambda}$ uma família limitada de funções de variação limitada em $[a, b]$. Podemos construir duas famílias de funções crescentes $\mathfrak{F}^{+}=\left\{\phi_{\lambda}^{+}\right\}$e $\mathfrak{F}^{-}=\left\{\phi_{\lambda}^{-}\right\}$, 
com $\phi_{\lambda}^{+}, \phi_{\lambda}^{-}$dadas como na Proposição 1.33. No entanto, não temos a garantia de limitação para essas famílias. Com a hipótese adicional de limitação uniforme em $\lambda$ para a variação total em $\mathfrak{F}$, teremos que $\mathfrak{F}^{+}, \mathfrak{F}^{-}$é uma decomposição de $\mathfrak{F}$ como famílias limitadas de funções crescentes. De fato, se $f$ é membro de $\mathfrak{F}$, portanto uma função de variação limitada em $[a, b]$, e $f^{+}, f^{-}$são como na Proposição 1.33, a equação (1.13) implica $f^{ \pm}(x) \leq f^{ \pm}(b)$ menor que $v_{f}(b)+|f(a)|$. Isto nos dá a limitaçāo superior. A limitação inferior é garantida pela limitação uniforme de $f$ em F. Os fatos anteriores serão usados para mostrarmos o resultado conhecido como Princípio da Seleção de Helly.

Teorema 1.38 (Princípio da Seleção de Helly). Seja $\mathfrak{F}=\left\{\phi_{\lambda}\right\}$ uma família infinita limitada de funções de variação limitada em $[a, b]$ com a variação total limitada uniformemente em $\lambda$. Então toda seqüência $\left(\phi_{n}\right)$ em $\mathfrak{F}$ admite uma subseqüência $\left(\phi_{n_{k}}\right)$ que converge pontualmente para uma função $\phi$ de variação limitada em $[a, b] e$, além disso,

$$
V(\phi,[a, b]) \leq \underline{\lim } V\left(\phi_{n_{k}},[a, b]\right) .
$$

Demonstração: Seja $\left(\phi_{n}\right)$ uma seqüência em $\mathfrak{F}$. Cada $\phi_{n}$ é decomposta, como na Proposição 1.33, em $\phi_{n}^{+}, \phi_{n}^{-}$, de modo que

$$
\phi_{n}(x)=\phi_{n}^{+}(x)-\phi_{n}^{-}(x) .
$$

As seqüências $\left(\phi_{n}^{+}\right),\left(\phi_{n}^{-}\right)$são limitadas (veja as considerações anteriores). Pelo Teorema $1.37,\left(\phi_{n}^{+}\right)$admite uma subseqüência $\left(\phi_{n_{k}}^{+}\right)$, convergente pontualmente em $[a, b]$. Seja agora a seqüência $\left(\phi_{n_{k}}^{-}\right)$. Novamente, esta seqüência admite subseqüência $\left(\phi_{n_{k_{i}}}^{-}\right)$ convergente em $[a, b]$. Renomearemos a seqüência $\left(\phi_{n_{k_{i}}}\right)$ novamente como $\left(\phi_{n_{k}}\right)$. Temos, então, que $\left(\phi_{n_{k}}\right)$ é subseqüência de $\left(\phi_{n}\right)$ convergente em todo $x$ de $[a, b]$ para a função $\phi$ dada por

$$
\phi(x)=\lim \left[\phi_{n_{k}}^{+}(x)\right]-\lim \left[\phi_{n_{k}}^{-}(x)\right],
$$

de variação limitada por ser diferença de funçōes crescentes.

A segunda parte do teorema segue do fato de, para uma partição $\pi: a=t_{0}<$ $t_{1}<\cdots<t_{N}=b$ de $[a, b]$,

$$
\sum_{i=1}^{N}\left|\phi\left(t_{i}\right)-\phi\left(t_{i-1}\right)\right|=\lim _{n_{k}} \sum_{i=1}^{N}\left|\phi_{n_{k}}\left(t_{i}\right)-\phi_{n_{k}}\left(t_{i-1}\right)\right| \leq \varliminf_{n_{k}} V\left(\phi_{n_{k}},[a, b]\right)
$$




\section{Continuidade Absoluta}

Definição 1.39. Uma funçāo $f:[a, b] \rightarrow \mathbb{R}$ é dita absolutamente contínua em $[a, b]$ se, para todo $\varepsilon>0$ dado, existir $\delta>0$ tal que

$$
\sum_{i=1}^{n}\left|f\left(b_{i}\right)-f\left(a_{i}\right)\right|<\varepsilon
$$

para toda coleção finita $\left\{\left(a_{i}, b_{i}\right)\right\}$ de subintervalos disjuntos de $[a, b]$ com

$$
\sum_{i=1}^{n}\left|b_{i}-a_{i}\right|<\delta
$$

É evidente que uma função absolutamente contínua é contínua. Uma função contínua não é necessariamente de variação limitada. Isto pode ser visto considerando a existência de funçōes contínuas que não são diferenciáveis em ponto algum, e portanto não podem ser de variação limitada. No entanto, o lema a seguir garante que a implicação é verdadeira se a continuidade for absoluta.

Lema 1.40. Se $f$ é absolutamente contínua em $[a, b]$, então $f$ é de variação limitada em $[a, b]$.

Demonstração: Seja $\delta>0$ como na definição 1.39, correspondente a $\varepsilon=1$. Fixemos $N \in \mathbb{N}$ tal que $\frac{b-a}{N}<\delta$. Seja $\pi=\left\{t_{0}, t_{1}, \ldots, t_{n}\right\}$ uma partição de $[a, b]$. Por um refinamento de $\pi$, obtemos uma partição $\tilde{\pi}=\left\{\tilde{t}_{0}, \tilde{t}_{1}, \ldots, \tilde{t}_{m}\right\}$ acrescentando a $\pi$ os pontos $\left\{a+\frac{k}{N}(b-a): 1 \leq k<N\right\}$. Como $\tilde{\pi}$ refina $\pi$, segue

$$
\sum_{i=1}^{n}\left|f\left(t_{i}\right)-f\left(t_{i-1}\right)\right| \leq \sum_{i=1}^{m}\left|f\left(\tilde{t}_{i}\right)-f\left(\tilde{t}_{i-1}\right)\right| \leq N
$$

pois $f$ é absolutamente contínua, e $\tilde{\pi}$ divide $[a, b]$ em $N$ coleções de intervalos cuja soma dos comprimentos dos intervalos de cada coleção é menor que $\delta$. Como $\pi$ foi tomada de modo arbitrário, segue $V(f,[a, b]) \leq N$.

Corolário 1.41. Se fé absolutamente contínua, então $f$ tem uma derivada definida em quase toda parte. 
Em geral, para uma função $f$ de variação limitada, não temos $f(x)=f(a)+$ $\int_{a}^{x} f^{\prime}(t) d t$, ou seja, $f$ não é a integral de sua derivada. Esta é uma propriedade reservada às funções absolutamente contínuas.

Definição 1.42 (Integral Indefinida). Uma função $F:[a, b] \rightarrow \mathbb{R}$ é dita uma integral indefinida se existir uma função $f$ definida em quase toda parte de $[a, b]$ tal que

$$
F(t)=F(a)+\int_{a}^{t} f(s) d s
$$

Teorema 1.43. Uma função $F$ é uma integral indefinida se, e somente se, $F$ é absolutamente contínua. Em outras palavras, se F é absolutamente contínua, então

$$
F(x)=F(a)+\int_{a}^{x} F^{\prime}(t) d t
$$

Veja Royden [6, Th. 5.13] para a demonstração destes fatos.

\section{Funções do Tipo $\varphi$}

Estaremos interessados numa subclasse das funções de variação limitada que sāo contínuas à direita e diferenciáveis por partes, devido à simplicidade da análise com estas funçōes e a representar o tipo dos impulsos, na maioria das aplicações. Nos próximos capítulos, restringir-nos-emos a estas funções.

Definição 1.44 (Função do Tipo $\varphi$ ). Uma função $u: J \rightarrow \mathbb{R}, J=\left[t_{0}, \infty\right)$, é dita do tipo $\varphi$ se

i. u é uma função contínua à direita e de variação limitada em todo subintervalo compacto de $J$

ii. O conjunto de pontos onde u é descontínua é finito ou enumerável e, neste caso, as descontinuidades $t_{1}<t_{2}<\cdots$ são tais que $t_{0}<t_{1} e$ não se acumulam em ponto algum, ou seja, $t_{k} \rightarrow \infty$ quando $k \rightarrow \infty$;

iii. $u$ é diferenciável em cada intervalo semi aberto onde u é contínua, entendendo-se a derivada no extremo esquerdo como a derivada lateral à direita.

Pela condição $i$, toda função do tipo $\varphi$ gera uma medida de Borel. 


\section{Distribuições}

Definição 1.45 (Suporte). Seja $f$ uma função contínua tomando valores em $\mathbb{R}^{n}$. Definimos o suporte de $f$, denotado $\operatorname{Supp}(f)$ como o fecho do conjunto

$$
\{x: f(x) \neq 0\}
$$

Definição $1.46\left(\mathcal{C}_{c}^{\infty}\right)$. Para um aberto $\Omega$ de $\mathbb{R}^{n}$, denotamos por $\mathcal{C}_{c}^{\infty}(\Omega)$ a coleção de todas as funções a valores em $\mathbb{R}$ de classe $\mathcal{C}^{\infty}$ definidas em $\Omega$ tendo suporte compacto. $\mathcal{C}_{c}^{\infty} e ́$ espaço vetorial normado, se tomarmos a norma do supremo em $\Omega$,

$$
\|\phi\|=\sup _{x \in \Omega}|\phi(x)|
$$

Definição 1.47 (Distribuição). Um funcional linear contínuo $T: \mathcal{C}_{c}^{\infty}(\Omega) \rightarrow \mathbb{R}$ é dito uma distribuição em $\Omega$. O espaço das distribuições em $\Omega$ é denotado $\mathcal{D}(\Omega)$. O espaço $\mathcal{D}(\Omega)$, sendo o espaço dual de $\mathcal{C}_{c}^{\infty}(\Omega)$, também é denotado como $\mathcal{C}_{c}^{\infty}(\Omega)^{\prime}$.

Por vezes, é conveniente escrevermos $\langle T, \phi\rangle$ em vez de $T(\phi)$. Vejamos alguns exemplos de distribuições.

Exemplo 1.48 (Delta de Dirac). Consideremos $\Omega=\mathbb{R}^{n}$ e $t_{0} \in \Omega$. Definamos $\langle\delta, \phi\rangle=$ $\phi\left(t_{0}\right)$ para $\phi \in \mathcal{C}_{c}^{\infty}$. Este funcional é claramente linear e contínuo. Esta distribuição é conhecida como Delta de Dirac concentrado no ponto $t_{0} e$ denotada por $\delta=\delta_{t_{0}}$.

Exemplo 1.49 (Medidas). Seja $\mu$ uma medida de Borel. Então $\mu(K)<\infty$ para todo compacto $K \subset \Omega$. O funcional

$$
\left\langle T_{\mu}, \phi\right)=\int_{\Omega} \phi d \mu
$$

é linear e contínuo, pela estimativa

$$
\left|\left\langle T_{\mu}, \phi\right)\right| \leq\|\phi(t)\| \cdot|\mu| \operatorname{Supp}(\phi) .
$$

Temos, então, que $T_{\mu}$ é uma distribuição.

Se $\alpha$ é uma função contínua à direita de variação limitada, a distribuiçāo dada pela medida de Borel da é denotada por $T_{d \alpha}$. 
Exemplo 1.50 (Funções Localmente Integráveis). Uma função $f \mu$-mensurável definida em $\Omega$ é dita localmente integrável se, para todo compacto $K \subset \Omega$,

$$
\int_{K} f d \mu<\infty
$$

Seja $f$ uma função localmente integrável. Definamos $T_{f}$ por

$$
\left\langle T_{f}, \phi\right\rangle=\int_{\Omega} \phi(t) f(t) d t
$$

$T_{f}$ define uma distribuição pois é claramente linear e a continuidade é dada por

$$
\left|\left\langle T_{f}, \phi\right\rangle\right| \leq\|\phi\| \cdot\left|\int_{\operatorname{Supp}(\phi)}\right| f(t)|d t| .
$$

Além disso, se $T_{f}=T_{g}$, então $f=g$ em quase toda parte. A demonstração deste fato pode ser encontrada em Hounie [3], Exemplo I.3.3.

Se $f$ é uma função localmente integrável, abandonemos a notação provisória $T_{f}$ e escrevamos simplesmente $\langle f, \phi\rangle=\int f \phi d t$. Isto equivale a identificar uma função localmente integrável $f$ com a distribuição $T_{f}$. Como a classe das funções localmente integráveis abrange os espaços $L p, 1 \leq p \leq \infty$, e $\mathcal{C}^{k}, 0 \leq k \leq \infty$, esta identificação permite-nos considerar $L^{p}$ e $\mathcal{C}^{k}$ como subespaços de $\mathcal{D}$. Neste sentido, podemos dizer que as distribuições são "funções generalizadas". Daqui em diante, a identificação $f \mapsto T_{f}$ será feita sem maiores comentários. Isto valerá também para a identificação da medida de Borel $\mu$ com a distribuição definida no exemplo 1.49.

Lema 1.51 (Produto por Funções). Se g é uma função integrável com respeito a $\mu$ e $T$ é uma distribuição em $\Omega$ dada por

$$
\langle T, \phi\rangle=\int_{\Omega} \phi d \mu, \quad \phi \in \mathcal{C}_{c}^{\infty}(\Omega)
$$

então o produto $g T$ definido por

$$
\langle g T, \phi\rangle=\int_{\Omega} \phi g d \mu, \quad \phi \in \mathcal{C}_{c}^{\infty}(\Omega)
$$

é também uma distribuição em $\Omega$.

Demonstração: Como $\phi$ pertence a $\mathcal{C}_{c}^{\infty}(\Omega)$, é limitada e $\mu$-integrável. Como $g$ é $\mu$-integrável, então o produto $\phi g$ é $\mu$-integrável. Logo, o segundo membro de (1.14) faz sentido. 
Claramente $g T$ é um funcional linear em $\bigodot_{c}^{\infty}(\Omega)$. Além disso,

$$
|\langle g T, \phi\rangle| \leq \int_{\Omega}|g||\phi| d|\mu| \leq\|\phi\| \int_{\Omega}|g| d|\mu| .
$$

Então $g T$ é um funcional contínuo em $\mathcal{C}_{c}^{\infty}$.

\subsection{Derivada Distribucional}

Consideremos $f: \mathbb{R} \rightarrow \mathbb{R}$ diferenciável e $\phi \in \mathcal{C}_{c}^{\infty}(\mathbb{R})$. Existe $M$ suficientemente grande tal que $\operatorname{Supp}(\phi) \subseteq[-M, M]$. Também, é fácil ver que $\operatorname{Supp}\left(\phi^{\prime}\right) \subseteq[-M, M]$. Então

$$
\begin{aligned}
\left\langle f^{\prime}, \phi\right\rangle & =\int_{\mathbb{R}} \phi(t) f^{\prime}(t) d t=\int_{-M}^{M} \phi(t) f^{\prime}(t) d t \\
& =\left.\phi(\cdot) f(\cdot)\right|_{-M} ^{M}-\int_{-M}^{M} \phi^{\prime}(t) f(t) d t \\
& =-\int_{\mathbb{R}} \phi^{\prime}(t) f(t) d t \\
& =-\left\langle f, \phi^{\prime}\right\rangle .
\end{aligned}
$$

No intuito de definirmos uma derivada para as distribuições, é natural impormos que a derivada da distribuição dada por $f$ seja a distribuição dada por $f^{\prime}$, visando a generalização de $\mathcal{C}^{1}(\mathbb{R})$ por $\mathcal{D}(\mathbb{R})$. Isto motiva-nos a seguinte definição para a derivada de uma distribuição.

Definição 1.52 (Derivada Distribucional). Sejam $\left(x^{1}, \ldots, x^{n}\right)$ coordenadas cartesianas em $\Omega$, e $T$ uma distribuição em $\Omega$. A derivada distribucional de $T$ com respeito a $x^{i}$, $1 \leq i \leq n$, denotada como $D_{i} T$, é definida por

$$
\left\langle D_{i} T, \phi\right\rangle=-\left\langle T, \frac{\partial \phi}{\partial x^{i}}\right\rangle, \quad \phi \in \mathcal{C}_{c}^{\infty} \Omega
$$

a qual, por sua vez, é uma distribuição em $\Omega$. Neste sentido, toda distribuição é infinitamente diferenciável.

Como uma função $f$ localmente integrável em um intervalo aberto de $\mathbb{R}$ pode ser identificada com a distribuição $T_{f}$, então $D T_{f}$ será denotada $D f$, e chamada a derivada distribucional de $f$. Quando $f$ é absolutamente contínua, portanto de 
variação limitada, o que valida a fórmula de integração por partes usada na motivação para a derivada distribucional, teremos que $D f$ é a derivada ordinária $f^{\prime}, f^{\prime}$ sendo identificada com a distribuição $T_{f^{\prime}}$. Se $f$ é de variação limitada e contínua à direita, então $D f$ é a medida $d f$ (gerada por $f$ ), sendo $d f$ identificada com a distribuição $T_{d f}$. Isto se deve ao fato de que toda função em $\bigodot_{c}^{\infty}$ é contínua e de variação limitada, tornando válida a fórmula de integração por partes dada no Corolário 1.35.

Vejamos o que ocorre com funções de uma variável que apresentam uma descontinuidade de primeira espécie na origem. Mais precisamente, suponhamos que $f \in \mathfrak{C}^{1}(\mathbb{R} \backslash\{0\})$ e que os limites $f(0+), f(0-)$ existam e são finitos. $f^{\prime}$ é localmente integrável, apesar de não estar definida na origem. Seja $\phi \in \mathcal{C}_{c}^{\infty}(\mathbb{R})$ e $M$ tal que $\operatorname{Supp}(\phi) \subseteq[-M, M]$. Calculemos $D f$.

$$
\begin{aligned}
\langle D f, \phi\rangle & =-\left\langle f, \phi^{\prime}\right\rangle=-\lim _{\varepsilon \rightarrow 0}\left(\int_{-M}^{-\varepsilon} f \phi^{\prime} d t+\int_{\varepsilon}^{M} f \phi^{\prime} d t\right) \\
& =\lim _{\varepsilon \rightarrow 0}\left(\left.f(\cdot) \phi(\cdot)\right|_{\varepsilon} ^{M}+\left.f(\cdot) \phi(\cdot)\right|_{-M} ^{-\varepsilon}\right)-\lim _{\varepsilon \rightarrow 0}\left(\int_{-M}^{-\varepsilon} f^{\prime} \phi d t+\int_{\varepsilon}^{M} f^{\prime} \phi d t\right) \\
& =[f(0+)-f(0-)] \phi(0)+\int_{-\infty}^{\infty} f^{\prime} \phi d t
\end{aligned}
$$

Trataremos, no próximo exemplo, da função contínua à direita de variação limitada não contínua mais simples: a função de Heaviside. Toda função do tipo $\varphi$ é decomposta como a soma de uma função contínua diferenciável por partes com uma função singular, formada por somas de funções múltiplas de $H\left(t-t_{k}\right)$. Daí a importância da função $H$.

Exemplo 1.53 (Heaviside). Seja $H$ a função de Heaviside, dada por

$$
H(x)=\left\{\begin{array}{ll}
0 & \text { se } x<0 \\
1 & \text { se } x \geq 0
\end{array} .\right.
$$

É claro que $H(0-)=0, H(0+)=1$ e $H^{\prime}=0$ em quase toda parte. Para $\phi \in \mathfrak{C}_{c}^{\infty}(\mathbb{R})$, temos

$$
\langle D H, \phi\rangle=\phi(0)=\left\langle\delta_{0}, \phi\right\rangle .
$$

Logo, a derivada distribucional da função de Heaviside é o Delta de Dirac concentrado na origem.

É usual denotarmos por $H_{t_{0}}(t):=H\left(t-t_{0}\right)$ a função de Heaviside com salto no instante $t_{0}$. 
Seguindo as considerações anteriores ao Exemplo 1.53, se $f$ é uma função do tipo $\varphi$ dada por

$$
f(t)=F(t)+\sum_{i=1}^{\infty} a_{k} H\left(t-t_{k}\right),
$$

sendo $F$ diferenciável em $J \backslash\left\{t_{k}\right\}_{k \in \mathbb{N}}$, podemos propor a fórmula

$$
D f=F^{\prime}+\left[f\left(t_{k}+\right)-f\left(t_{k}-\right)\right] \delta_{k} .
$$




\section{Capítulo 2}

\section{Existência e Unicidade}

\section{Equações Diferenciais em Medida}

Sejam $F, G$ funções contínuas definidas em $J \times \mathbb{R}^{n}$ com valores em $\mathbb{R}^{n}$, sendo $J=$ $\left[T_{0}, \infty\right)$, e $u$ uma função real contínua à direita de variação limitada em $J$. Seja $\mathcal{S}$ um aberto conexo em $\mathbb{R}^{n}$ e $I$ um intervalo com extremo inferior $t_{0} \geq T_{0}$.

Considere então a Equação Diferencial em Medida (EDM)

$$
D x=F(t, x)+G(t, x) D u
$$

onde a igualdade é tomada em $\mathcal{D}(\mathcal{S})$, ou seja, para cada $\phi \in \mathfrak{C}_{c}^{\infty}(\mathcal{S})$,

$$
\int_{\mathcal{S}} \phi(t) d x(t)=\int_{\mathcal{S}} \phi(t) F(t, x(t)) d t+\int_{\mathcal{S}} \phi(t) G(t, x(t)) d u(t) .
$$

Definição 2.1 (Solução de uma EDM). Uma função $x(\cdot)$ é dita uma solução da EDM (2.1) por $\left(t_{0}, x_{0}\right)$ em I se $x$ é uma função contínua à direita de variação limitada satisfazendo $x\left(t_{0}\right)=x_{0}$ e a derivada distribucional de $x$ satisfaz a equação $(2.1)$ em $\left(t_{0}, \tau\right)$ para todo $\tau$ em $I$.

Exemplo 2.2. Considere a equação em medida

$$
D x=1+D H, \quad x(0)=0
$$

sendo $H$ a funçāo de Heaviside. Sua única solução é

$$
x(t)=t+H(t)-1 \text {. }
$$


De fato, sejam $\hat{x}$ e $\breve{x}$ soluções distintas de (2.2). Como o segundo membro de (2.2) independe de $x$, temos $D(\hat{x}-\check{x})=0$, a distribuição nula. Para toda $\phi \in \mathcal{C}_{c}^{\infty}(\mathbb{R})$, temos

$$
0=(D(\hat{x}-\check{x}), \phi\rangle=-\left(\hat{x}-\check{x}, \phi^{\prime}\right\rangle \text {. }
$$

Devemos, entāo, caracterizar as funçōes $\varphi \in \mathcal{C}_{c}^{\infty}(\mathbb{R})$ que possuem primitiva em $\mathcal{C}_{c}^{\infty}(\mathbb{R})$. Se $\phi, \varphi \in \mathcal{C}_{c}^{\infty}(\mathbb{R})$ satisfazem $\varphi=\phi^{\prime}$, então $\int_{\mathbb{R}} \varphi=0$, pois $\phi(-M)=\phi(M) \doteq 0$ sempre que $\operatorname{Supp}(\phi) \subseteq[-M, M]$. Reciprocamente, se $\varphi \in \mathcal{C}_{c}^{\infty}(\mathbb{R}) \operatorname{satisfaz} \operatorname{Supp}(\varphi) \subseteq$ $[-M, M] e \int_{\mathbb{R}} \varphi=0$, então $\phi$, definida como $\phi(t)=0$ para $t$ fora de $[-M, M] e$ $\phi(t)=\int_{-M}^{t} \varphi$ para $t \in[-M, M]$, é um elemento de $\mathcal{C}_{c}^{\infty}(\mathbb{R})$ e claramente $\varphi=\phi^{\prime}$. Logo, $\varphi \in \mathcal{C}_{c}^{\infty}(\mathbb{R})$ admite uma primitiva em $\mathcal{C}_{c}^{\infty}(\mathbb{R})$ se, e somente se, $\int_{\mathbb{R}} \varphi=0$.

Seja $\phi \in \mathcal{C}_{c}^{\infty}(\mathbb{R})$. Existe uma funçāo $\rho \in \mathcal{C}_{c}^{\infty}(\mathbb{R})$ tal que $\operatorname{Supp}(\rho) \subseteq[-1,1] e$ $\int_{-1}^{1} \rho=1$. Então a função

$$
\varphi_{\phi}(t)=\phi(t)-\rho(t) \int_{\mathbf{R}} \phi
$$

é elemento de $\mathcal{C}_{c}^{\infty}(\mathbb{R}) e \int_{\mathbb{R}} \varphi_{\phi \mathrm{l}}=0$, ’́portanto $\varphi_{\phi}$ admite uma primitiva em $\mathrm{C}_{c}^{\infty}(\mathbb{R})$. Logo, por (2.3), temos

$$
(\hat{x}-\check{x}, \phi\rangle=(\hat{x}-\check{x}, \rho\rangle \int_{\mathbb{R}} \phi=C \int_{\mathbb{R}} \phi,
$$

$\operatorname{com} C=\langle\hat{x}-\check{x}, \rho\rangle$. Então $\hat{x}-\check{x}=C$, no sentido das distribuiçōes. Como no Exemplo 1.50, $\hat{x}-\check{x}=C$ em quase toda parte, no sentido usual. Isto quer dizer que, para todo $t$, existe uma sequência $\left(t_{n}\right), t_{n}>t$ tal que $[\hat{x}-\check{x}]\left(t_{n}\right)=C$. Como $\hat{x}-\check{x}$ é contínua à direita, concluímos $[\hat{x}-\check{x}](t)=C$ para todo $t \in \mathbb{R}$, mas $[\hat{x}-\check{x}](0)=0$. Esta mostrada a unicidade da solução apresentada.

\section{Representação Integral}

Propriedades qualitativas de soluções de equações diferenciais podem ser melhor estudadas convertendo-se a equação diferencial em uma equação integral. É de fácil verificação que o p.v.i. ordinário

$$
\dot{x}=f(t, x), \quad x\left(t_{0}\right)=x_{0}
$$

é equivalente à equação integral

$$
x(t)=x_{0}+\int_{t_{0}}^{t} f(s, x(s)) d s .
$$


Para as equações diferenciais em medida, temos um resultado similar, porém não mais de verificação trivial.

Teorema 2.3 (Representação Integral). $x(\cdot)$ é uma solução de (2.1) por $\left(t_{0}, x_{0}\right)$ em I se e somente se $x(\cdot)$ satisfaz a equação integral

$$
x(t)=x_{0}+\int_{t_{0}}^{t} F(s, x(s)) d s+\int_{t_{0}}^{t} G(s, x(s)) d u(s), \quad t \in I,
$$

lembrando que a segunda integral $\int_{t_{0}}^{t} G(s, x(s)) d u(s)$ é considerada sobre o intervalo semi-aberto $\left(t_{0}, t\right]$.

Demonstração: Suponha que $x(\cdot)$ satisfaça a equação (2.4) para $t \in I$. Então as integrais $\int_{t_{0}}^{t} F(s, x(s)) d s$ e $\int_{t_{0}}^{t} G(s, x(s)) d u(s)$ estão definidas para $t \in I$.

A integral $\int_{t_{0}}^{t} F(s, x(s)) d s$ é uma função absolutamente contínua por ser a integral indefinida da função mensurável $F(\cdot, x(\cdot))$. Logo, é contínua e de variação limitada em $r$.

Afirmamos que a integral $\int_{t_{0}}^{t} G(s, x(s)) d u(s)$ é uma função de variação limitada em $I$. De fato, como $G(\cdot, x(\cdot))$ é $d u$-mensurável, segue que $|G(\cdot, x(\cdot))|$ também é. Fixemos $t \in I$. Se $\pi: t_{0}=\tau_{0}<\tau_{1}<\cdots<\tau_{n}=t$ é uma partição do intervalo $\left[t_{0}, t\right]$, temos

$$
\begin{aligned}
& \sum_{i=i}^{n}\left|\int_{\left(t_{0}, \tau_{i}\right]} G(s, x(s)) d u(s)-\int_{\left(t_{0}, \tau_{i-1}\right]} G(s, x(s)) d u(s)\right| \\
= & \sum_{i=i}^{n}\left|\int_{\left(\tau_{i-1}, \tau_{i}\right]} G(s, x(s)) d u(s)\right| \\
\leq & \sum_{i=i}^{n} \int_{\left(\tau_{i-1}, \tau_{i}\right]}|G(s, x(s))| d v_{u}(s) \\
= & \int_{\left(t_{0}, t\right]}|G(s, x(s))| d v_{u}(s) .
\end{aligned}
$$

Além disso, $\int_{t_{0}}^{t} G(s, x(s)) d u(s)$ é contínua à direita. De fato, seja $\left(\tau_{n}\right) \rightarrow t$ uma seqüência tal que $\tau_{n}>t$ e $\tau_{1} \geq \tau_{n}$ para todo $n$. Temos que $d u\left(t, \tau_{n}\right] \rightarrow 0$ por ser $\left\{\left(t, \tau_{n}\right]\right\}_{n \in \mathbb{N}}$ uma cadeia de intervalos encaixantes e $\bigcap\left(t, \tau_{n}\right]=\varnothing$. Se $\varphi=\sum_{i=1}^{k} c_{k} \chi_{E_{k}}$ é uma função simples em $\left(t, \tau_{1}\right]$ tal que $\varphi(s) \leq G(s, x(s))$, então

$$
\int_{\left(t, \tau_{n}\right]} \varphi(s) d u(s)=\sum_{i=1}^{k} c_{k} d \tilde{u}\left(E_{k} \cap\left(t, \tau_{n}\right]\right) \rightarrow 0 \quad \text { quando } n \rightarrow \infty .
$$


Como isto vale para toda função simples nas condições acima, segue que

$$
\int_{\left(t, \tau_{n}\right]} G(s, x(s)) d u(s) \rightarrow 0 \quad \text { quando } n \rightarrow \infty
$$

Logo $x(\cdot)$ é uma função contínua à direita e de variação limitada. Claramente, $x\left(t_{0}\right)=x_{0}$.

Se $\tau$ é um ponto arbitrário em $I$ e $x^{i}(\cdot)$ é a $i$-ésima componente de $x(\cdot)$ em $I_{\tau}=\left(t_{0}, \tau\right)$, então

$$
\left\langle x^{i}, \phi\right\rangle=\int_{I_{\tau}}\left\{x_{0}^{i}+\int_{t_{0}}^{t} F^{i}(s, x(s)) d s+\int_{t_{0}}^{t} G^{i}(s, x(s)) d u(s)\right\} \phi(t) d t
$$

para $\phi \in \mathcal{C}_{c}^{\infty}\left(I_{\tau}\right)$. Portanto

$$
\begin{aligned}
\left\langle D x^{i}, \phi\right\rangle & =-\left\langle x^{i}, \phi^{\prime}\right\rangle \\
& =-\int_{I_{\tau}}\left\{x_{0}^{i}+\int_{t_{0}}^{t} F^{i}(s, x(s)) d s+\int_{t_{0}}^{t} G^{i}(s, x(s)) d u(s)\right\} \phi^{\prime}(t) d t
\end{aligned}
$$

Agora,

$$
-\int_{I_{\tau}}\left\{x_{0}^{i}+\int_{t_{0}}^{t} F^{i}(s, x(s)) d s\right\} \phi^{\prime}(t) d t=\int_{I_{\tau}} F^{i}(s, x(s)) \phi(s) d s
$$

integrando por partes e usando $\phi\left(t_{0}\right)=0=\phi(\tau)$.

Seja $g(t)=\int_{t_{0}}^{t} G^{i}(s, x(s)) d u(s)$. Então $g(\cdot)$ é contínua à direita e de variação limitada em $I_{\tau}$. O Teorema 1.19 nos dá

$$
\begin{aligned}
\int_{I_{\tau}} g(t) \phi^{\prime}(t) d t & =\int_{\left(t_{0}, \tau\right)} g(t) d \phi(t) \\
& =\int_{\left(t_{0}, \tau\right]} g(t) d \phi(t)-\int_{\{\tau\}} g(t) d \phi(t)
\end{aligned}
$$

Mas, como $\phi$ é contínua, $\int_{\{\tau\}} g(t) d \phi(t)=0$ e, pelo Corolário 1.35,

$$
\begin{aligned}
\int_{\left(t_{0}, \tau\right)} g(t) d \phi(t) & =g(\tau) \phi(\tau)-g\left(t_{0}\right) \phi\left(t_{0}\right)-\int_{\left(t_{0}, \tau\right)} \phi(t) d g(t) \\
& =-\int_{I_{\tau}} \phi(t) d g(t) \\
& =-\int_{\left(t_{0}, \tau\right)} \phi(t) G^{i}(t, x(t)) d u(t)
\end{aligned}
$$


Das equações (2.5) a (2.7), obtemos

$$
\left\langle D x^{i}, \phi\right\rangle=\int_{I \tau} \phi(t) F^{i}(t, x(t)) d t+\int_{I_{\tau}} \phi(t) G^{i}(t, x(t)) d u(t)
$$

A segunda distribuição no segundo membro de (2.8) é, pelo lema 1.51, identificada com a medida dada por $G^{i}(t, x(t)) d u(t)$, enquanto a primeira distribuição é identificada com $F^{i}(t, x(t))$. Como isto vale para $i=1,2, \ldots, n$, a derivada $D x$ é identificada com $F(t, x(t))+G(t, x(t)) D u$ e, portanto, $x$ é solução de (2.1) por $\left(t_{0}, x_{0}\right)$.

Reciprocamente, suponhamos $x$ solução de (2.1) por $\left(t_{0}, x_{0}\right)$ em $I$. Então, para $I_{\tau}=\left(t_{0}, \tau\right)$, sendo $\tau$ um ponto arbitrário de $I$, temos

$$
\int_{I_{\tau}} \phi(t) d x^{i}(t)=\int_{I_{\tau}} F^{i}(t, x(t)) d t+\int_{I_{\tau}} G^{i}(t, x(t)) d u(t)
$$

para $i=1,2, \ldots, n$ e para cada $\phi \in \mathcal{C}_{c}^{\infty}\left(I_{\tau}\right)$.

Integrando o primeiro membro de (2.9) por partes e usando (2.6) e (2.7), obtemos

$$
\begin{aligned}
& \int_{I_{\tau}} \phi^{\prime}(t)\left(x^{i}(t)-x_{0}^{i}\right) d t \\
&=\int_{I_{\tau}} \phi^{\prime}(t)\left\{\int_{t_{0}}^{t} F^{i}(s, x(s)) d s+\int_{t_{0}}^{t} G^{i}(s, x(s)) d u(s)\right\} d t .
\end{aligned}
$$

Isto implica

$$
x^{i}(t)=x_{0}^{i}+\int_{t_{0}}^{t} F^{i}(s, x(s)) d s+\int_{t_{0}}^{t} G^{i}(s, x(s)) d u(s)
$$

em quase toda parte em $I_{\tau}$. No entanto, como $x$ é contínua à direita (como solução de (2.1)), e como o segundo membro de (2.11) é uma função contínua à direita em $t$, a igualdade em (2.11) é satisfeita em toda parte de $I_{\tau}$. Então $x$ satisfaz (2.4) para todo $t$ em $I$.

\section{Existência de Soluções}

Sejam $\mathcal{S} \subseteq \mathbb{R}^{n}$ um aberto conexo e $I$ um intervalo com extremo inferior $t_{0} \geq T_{0}$. Definamos

$$
\begin{array}{r}
\mathbb{B}\left(x_{0}, b\right)=\left\{x \in \mathbb{R}^{n}:\left|x-x_{0}\right|<b\right\} \\
E=\left[t_{0}, \hat{t}\right] \times \mathbb{B}\left(x_{0}, b\right)
\end{array}
$$


sendo $x_{0} \in \mathcal{S}$ e $b>0$ suficientemente pequeno para que $\mathbb{B}\left(x_{0}, b\right) \subseteq \mathcal{S}$.

Teorema 2.4 (Existência Local). Sejam $F$ e G satisfazendo às seguintes condições em $E$ :

i. A função $F(t, x)$ é mensurável em $t$ para cada $x$ e contínua em $x$ para cada $t$.

ii. A função $G(t, x)$ é contínua em $x$ para cada $t$ e $G(t, y(t))$ é du-integrâvel para cada $y \in B V\left(\left[t_{o}, \hat{t}\right], \mathbb{B}\left(x_{0}, b\right)\right)$.

iii. Existe uma função Lebesgue-integrável $f$ tal que

$$
|F(t, x)| \leq f(t) \quad \text { para }(t, x) \in E \text {. }
$$

iv. Existe uma funçāo dv $v_{u}$-integrâvel $g$ tal que

$$
|G(t, x)| \leq g(t) \quad \text { para }(t, x) \in E
$$

Entāo existe uma soluçāo $x(\cdot)$ de (2.1) em algum intervalo $\left[t_{0}, t_{0}+a\right]$ satisfazendo a condiçāo inicial $x\left(t_{0}\right)=x_{0}$.

Demonstração: Escolhamos $a$ tal que $0<2 a<\hat{t}-t_{0} \mathrm{e}$

$$
\int_{t_{0}}^{t_{0}+2 a} f(s) d s+\int_{t_{0}}^{t_{0}+2 a} g(s) d v_{u}(s) \leq c
$$

com $0<c<b$. Tal escolha é possível por

$$
\int_{t_{0}}^{t} f(s) d s \quad \text { e } \int_{t_{0}}^{t} g(s) d v_{u}(s)
$$

serem, respectivamente, contínua e contínua à direita em $t$.

Considere a equação integral

$x_{k}(t)= \begin{cases}x_{0}, & t \in\left[t_{0}-\frac{2 a}{k}, t_{0}\right], \\ x_{0}+\int_{t_{0}}^{t} F\left(s, x_{k}\left(s-\frac{2 a}{k}\right)\right) d s+\int_{t_{0}}^{t} G\left(s, x_{k}\left(s-\frac{2 a}{k}\right)\right) d u(s), & t \in\left(t_{0}, t_{0}+2 a\right] .\end{cases}$

Para todo $k$, a expressão (2.13) define $x_{k}$ em $\left[t_{0}-2 a / k_{\diamond} t_{0}+2 a\right]$ como uma função de variação limitada por $b$, isto é, $V\left(x_{k},\left[t_{0}-2 a / k, t_{0}+2 a\right]\right)<b$. De fato, $x_{k}$, restrita ao intervalo $\left[t_{0}-2 a / k, t_{0}\right]$, é constante de valor igual a $x_{0}$, portanto de 
variação nula. Suponha agora que $V\left(x_{k},\left[t_{0}, t_{0}+2 a j / k\right]\right)<b$. Como $x_{k}\left(t_{0}\right)=x_{0}$, teremos $\left|x_{k}(t)-x_{0}\right|<b$ para $t \in\left[t_{0}-2 a / k, t_{0}+2 a j / k\right]$. Se $\pi: t_{0}=\tau_{0}<\tau_{1}<\cdots<$ $\tau_{n}=t_{0}+2 a(j+1) / k$ é uma partição de $\left[t_{0}, t_{0}+2 a(j+1) / k\right]$, fazendo

$$
y(t)=x_{0}+\int_{t_{0}}^{t} F(s, x(s-2 a / k)) d s+\int_{t_{0}}^{t} G(s, x(s-2 a / k)) d u(s),
$$

então

$$
\begin{aligned}
& \sum_{i=1}^{n}\left|y\left(\tau_{i}\right)-y\left(\tau_{i-1}\right)\right| \\
= & \sum_{i=1}^{n}\left|\int_{\tau_{i-1}}^{\tau_{i}} F\left(s, x\left(s-\frac{2 a}{k}\right)\right) d s+\int_{\tau_{i-1}}^{\tau_{i}} G\left(s, x\left(s-\frac{2 a}{k}\right)\right) d u(s)\right| \\
\leq & \sum_{i=1}^{n} \int_{\tau_{i-1}}^{\tau_{i}}\left|F\left(s, x\left(s-\frac{2 a}{k}\right)\right)\right||d s|+\int_{\tau_{i-1}}^{\tau_{i}}\left|G\left(s, x\left(s-\frac{2 a}{k}\right)\right)\right||d u(s)| \\
\leq & \int_{t_{0}}^{t_{0}+2 a(j+1) / k} f(s) d s+\int_{t_{0}}^{t_{0}+2 a(j+1) / k} g(s) d v_{u}(s) \\
\leq & c<b .
\end{aligned}
$$

Como a partição $\pi$ é arbitrária, segue $V\left(x_{k},\left[t_{0}, t_{0}+2 a(j+1) / k\right]\right) \leq c<b$. Por $j$ também ser genérico, segue que $V\left(x_{k},\left[t_{0}-2 a / k, t_{0}+2 a\right]\right) \leq c<b$.

Além disso, $x_{k}$ é contínua à direita pelo mesmo argumento utilizado na prova do Teorema 2.3 quando mostramos que $\int_{t_{0}}^{t} G(s, x(s)) d u(s)$ é contínua à direita.

Definamos

$$
\tilde{x}_{k}(t)=x_{k}(t-2 a / k), \quad t \in\left[t_{0}-2 a / k, t_{0}+2(1-1 / k) a\right], \quad k \geq 2 .
$$

Como $x_{k}$ é contínua à direita, $\tilde{x}_{k}$ também é. Também, a família $\left\{\tilde{x}_{k}\right\}_{k \in \mathbb{N}}$ é de variação limitada uniformemente por $c$ no intervalo $\left[t_{0}, t_{0}+a\right]$. Pelo Princípio da Seleção de Helly (Teorema 1.38), existem uma subseqüência $\left(\tilde{x}_{k_{j}}\right)$ de $\left(\tilde{x}_{k}\right)$ e uma função $x^{\star}$ de variação limitada tais que

$$
\lim _{j \rightarrow \infty} \tilde{x}_{k_{j}}(t)=x^{\star}(t), \quad t \in\left[t_{0}, t_{0}+a\right] .
$$

A continuidade de $F$ e $G$ em $x$ implicam

$$
\begin{gathered}
\lim _{j \rightarrow \infty} F\left(t, \tilde{x}_{k_{j}}(t)\right)=F\left(t, x^{\star}(t)\right), \\
\lim _{j \rightarrow \infty} G\left(t, \tilde{x}_{k_{j}}(t)\right)=G\left(t, x^{\star}(t)\right) .
\end{gathered}
$$


Portanto, o Teorema da Convergência Dominada de Lebesgue nos dá

$$
\lim _{j \rightarrow \infty} \int_{t_{0}}^{t} F\left(s, \tilde{x}_{k_{j}}(s)\right) d s=\int_{t_{0}}^{t} F\left(s, x^{\star}(s)\right) d s
$$

$\mathrm{e}$

$$
\begin{aligned}
\lim _{j \rightarrow \infty} \int_{t_{0}}^{t} G\left(s, \tilde{x}_{k_{j}}(s)\right) d u(s) & =\lim _{j \rightarrow \infty}\left\{\int_{t_{0}}^{t} G\left(s, \tilde{x}_{k_{j}}(s)\right) d u^{+}(s)-\int_{t_{0}}^{t} G\left(s, \tilde{x}_{k_{j}}(s)\right) d u^{-}(s)\right\} \\
& =\int_{t_{0}}^{t} G\left(s, x^{\star}(s)\right) d u^{+}(s)-\int_{t_{0}}^{t}-G\left(s, x^{\star}(s)\right) d u^{-}(s) \\
& =\int_{t_{0}}^{t} G\left(s, x^{\star}(s)\right) d u(s),
\end{aligned}
$$

onde $d u^{+}, d u^{-}$denotam a variação positiva e negativa da medida de Borel-Stieltjes $d u$, respectivamente.

De (2.13), temos

$\tilde{x}_{k_{j}}(t+2 a / k)= \begin{cases}x_{0}, & t \in\left[t_{0}-\frac{2 a}{k}, t_{0}\right], \\ x_{0}+\int_{t_{0}}^{t} F\left(s, \tilde{x}_{k_{j}}(s)\right) d s+\int_{t_{0}}^{t} G\left(s, \tilde{x}_{k_{j}}(s)\right) d u(s), & t \in\left(t_{0}, t_{0}+a\right] .\end{cases}$

Tomando o limite quando $k \rightarrow \infty$, obtemos

$$
x^{\star}(t)=x^{\star}(t+)=x_{0}+\int_{t_{0}}^{t} F\left(s, x^{\star}(s)\right) d s+\int_{t_{0}}^{t} G\left(s, x^{\star}(s)\right) d u(s),
$$

para $t \in\left[t_{0}, t_{0}+a\right]$, por $x^{\star}$ ser contínua à direita. Logo, pelo Teorema $2.3, x^{\star}$ é solução de (2.1) por $\left(t_{0}, x_{0}\right)$ em $\left[t_{0}, t_{0}+a\right]$.

\section{Unicidade}

$O$ fato de $u$ poder ser descontínua dificulta o estabelecimento da unicidade de soluções de (2.1). O exemplo seguinte mostra que as soluções de (2.1) por um ponto não são necessariamente únicas mesmo quando $F$ e $G$ são lineares em $x$.

Exemplo 2.5. Considere a equação escalar

$$
D x=2(t+1)^{-1} x D u, \quad t \in[0,2],
$$

sendo $u(t)=t+H_{1}(t), \quad H_{1}$ denotando a função de Heaviside com salto em $t=1$. 
Pelo teorema 2.3, basta verificarmos que a expressāo

$$
x(t)=\left\{\begin{array}{cc}
0, & \text { para } 0 \leq t<1 \\
\frac{c}{4}(t+1)^{2}, & \text { para } 1 \leq t \leq 2
\end{array},\right.
$$

com c arbitrário, fornece uma infinidade de soluções de (2.14) por $(0,0)$ em [0,2].

Entretanto, se $u$ é do tipo $\varphi$ e certas condições adicionais são assumidas sobre $F$ e $G$, é possível obtermos unicidade para os p.v.i. associados a (2.1). Iniciemos nosso estudo mostrand o o clássico Lema de Gronwall para equações diferenciais ordinárias, visando sua extensão para equações em medida.

Lema 2.6 (Gronwall). Sejam $u$, $v$ funções contínuas não negativas em $[a, b]$ tais que, para $\alpha \geq 0$,

$$
u(t) \leq \alpha+\int_{a}^{t} v(s) u(s) d s, \quad t \in[a, b] .
$$

Então

$$
u(t) \leq \alpha \exp \left\{\int_{a}^{t} v(s) d s\right\} .
$$

Em particular, se $\alpha=0$, então $u \equiv 0$.

Demonstração: $\quad$ Se $\alpha>0$, para $\omega(t)=\alpha+\int_{a}^{t} v(s) u(s) d s$, temos $\omega(a)=\alpha \geq u(a)$ e $\omega(t) \geq \alpha>0$. De $\omega^{\prime}(t)=v(t) u(t) \leq v(t) \omega(t)$, tiramos $\omega^{\prime}(t) / \omega(t) \leq v(t)$. Integrando entre $a$ e $t$, obtemos

$$
\frac{\omega(t)}{\alpha} \leq \exp \left\{\int_{a}^{t} v(s) d s\right\}
$$

que nos dá

$$
u(t) \leq \omega(t) \leq \alpha \exp \left\{\int_{a}^{t} v(s) d s\right\} .
$$

Se $\alpha=0$, o caso anterior implica $u(t) \leq \hat{\alpha} \exp \left\{\int_{a}^{t} v(s) d s\right\}$ para todo $\hat{\alpha}>0$. Logo $u \equiv 0$.

O Teorema 2.7 é inspirado no Teorema de Pandit-Deo [5, Th. 2.3]. Na verdade, o Teorema de [5] pode ser obtido como corolário do Teorema 2.7.

Teorema 2.7 (Lema de Gronwall para EDM). Suponha que

i. As funções $x, g$ são nāo negativas, definidas e du-integraveis em $J$, e a funçāo $f$ é näo negativa, definida e integravel em $J$; 
ii. A função u é do tipo $\varphi$, sua derivada $u^{\prime}$ é nāo negativa em $J \backslash\left\{t_{k}\right\}$ e, além disso, os saltos $a_{k}=u\left(t_{k}\right)-u\left(t_{k}-\right)$ de $u$ em $t_{k}$ são tais que

$$
\begin{aligned}
& \left|a_{k}\right| g\left(t_{k}\right)<1, k=1,2, \ldots, \\
& P=\prod_{k=1}^{\infty}\left(1-\left|a_{k}\right| g\left(t_{k}\right)\right) \neq 0 .
\end{aligned}
$$

Nestas condições, para toda constante positiva c, a desigualdade

$$
x(t) \leq c+\int_{t_{0}}^{t} f(s) x(s) d s+\int_{t_{0}}^{t} g(s) x(s) d u(s), \quad t \in J
$$

implica

$$
x(t) \leq c P^{-1} \exp \left\{\int_{t_{0}}^{t}\left[f(s)+g(s) u^{\prime}(s)\right] d s\right\}, \quad t \in J
$$

Demonstração: Denotemos o segundo membro de (2.17) por $r(t)$. Como $u$ é diferenciável em $\left[t_{0}, t_{1}\right)$, e

$$
\begin{aligned}
r(t) & =c+\int_{t_{0}}^{t} f(s) x(s) d s+\int_{t_{0}}^{t} g(s) x(s) d u(s) \\
& \leq c+\int_{t_{0}}^{t} f(s) r(s) d s+\int_{t_{0}}^{t} g(s) r(s) d u(s)
\end{aligned}
$$

o Lema 2.6 nos dá

$$
r(t) \leq c \exp \left\{\int_{t_{0}}^{t}\left[f(s)+g(s) u^{\prime}(s)\right] d s\right\}, \quad t \in\left[t_{0}, t_{1}\right) .
$$

Em $t_{1}$, para $h>0$ suficientemente pequeno, de (2.18) e (2.19), obtemos

$$
\begin{aligned}
r\left(t_{1}\right) & =r\left(t_{1}-h\right)+\int_{t_{1}-h}^{t_{1}} f(s) x(s) d s+\int_{t_{1}-h}^{t_{1}} g(s) x(s) d u(s) \\
\leq & \operatorname{cexp}\left\{\int_{t_{0}}^{t_{1}-h}\left[f(s)+g(s) u^{\prime}(s)\right] d s\right\} \\
& \quad+\left|\int_{t_{1}-h}^{t_{1}} f(s) r(s) d s+\int_{t_{1}-h}^{t_{1}} g(s) r(s) d u(s)\right|
\end{aligned}
$$

Afirmamos que

$$
\lim _{h \rightarrow 0+}\left|\int_{t_{1}-h}^{t_{1}} f(s) r(s) d s+\int_{t_{1}-h}^{t_{1}} g(s) r(s) d u(s)\right|=\left|a_{1}\right| g\left(t_{1}\right) r\left(t_{1}\right) .
$$


De fato, consideremos a função de conjuntos $\lambda$ definida por

$$
\lambda(A)=\left|\int_{A} f(s) r(s) d s+\int_{A} g(s) r(s) d u(s)^{s}\right| .
$$

Sejam $\left(h_{k}\right)$ uma seqüência decrescente de números reais positivos tendendo a zero e $A_{k}=\left[t_{1}-h_{k}, t_{1}\right]$. Então $A_{1} \supset A_{2} \supset \cdots$ e $\bigcap_{k=1}^{\infty} A_{k}=\left\{t_{1}\right\}$. Portanto, o Teorema de Convergência Dominada de Lebesgue nos dá que $\lambda\left(A_{k}\right) \rightarrow \lambda\left(\left\{t_{1}\right\}\right)=\left|a_{1}\right| g\left(t_{1}\right) r\left(t_{1}\right)$, ficando (2.21) estabelecida. Tomando o limite quando $h \rightarrow 0+$ em (2.20), obtemos

$$
r\left(t_{1}\right) \leq c \exp \left\{\int_{t_{0}}^{t_{1}}\left[f(s)+g(s) u^{\prime}(s)\right] d s\right\}+\left|a_{1}\right| g\left(t_{1}\right) r\left(t_{1}\right)
$$

que, por (2.15), nos dá

$$
r\left(t_{1}\right) \leq c P_{1}^{-1} \exp \left\{\int_{t_{0}}^{t_{1}-h}\left[f(s)+g(s) u^{\prime}(s)\right] d s\right\}
$$

com

$$
P_{k}=\prod_{i=1}^{k}\left(1-\left|a_{i}\right| g\left(t_{i}\right)\right) \leq 1
$$

A seguir usaremos sucessivamente as desigualdades $1 \leq P_{1}^{-1} \leq P_{2}^{-1} \leq \cdots \leq P^{-1}$.

Para $t \in\left[t_{1}, t_{2}\right)$, notemos que

$$
\begin{aligned}
r(t) & =r\left(t_{1}\right)+\int_{t_{1}}^{t} f(s) x(s) d s+\int_{t_{1}}^{t} g(s) x(s) d u(s) \\
& \leq r\left(t_{1}\right)+\int_{t_{1}}^{t} f(s) r(s) d s+\int_{t_{1}}^{t} g(s) r(s) d u(s) .
\end{aligned}
$$

Como anteriormente, isto nos dá

$$
\begin{aligned}
r(t) & \leq r\left(t_{1}\right) \exp \left\{\int_{t_{1}}^{t}\left[f(s)+g(s) u^{\prime}(s)\right] d s\right\} \\
& \leq c P_{1}^{-1} \exp \left\{\int_{t_{0}}^{t}\left[f(s)+g(s) u^{\prime}(s)\right] d s\right\}, \quad t \in\left[t_{0}, t_{2}\right),
\end{aligned}
$$

por (2.22) e (2.23). Novamente, para $h$ pequeno,

$$
\begin{aligned}
& r\left(t_{2}\right)= r\left(t_{2}-h\right)+\int_{t_{2}-h}^{t_{2}} f(s) x(s) d s+\int_{t_{2}-h}^{t_{2}} g(s) x(s) d u(s) \\
& \leq c P_{1}^{-1} \exp \left\{\int_{t_{0}}^{t_{2}-h}\left[f(s)+g(s) u^{\prime}(s)\right] d s\right\} \\
&+\left|\int_{t_{2}-h}^{t_{2}} f(s) r(s) d s+\int_{t_{2}-h}^{t_{2}} g(s) r(s) d u(s)\right|
\end{aligned}
$$


que implica

$$
r\left(t_{2}\right)=c P_{1}^{-1} \exp \left\{\int_{t_{0}}^{t_{2}}\left[f(s)+g(s) u^{\prime}(s)\right] d s\right\}+\left|a_{2}\right| g\left(t_{2}\right) r\left(t_{2}\right),
$$

e portanto

$$
r\left(t_{2}\right) \leq c P_{2}^{-1} \exp \left\{\int_{t_{0}}^{t_{2}}\left[f(s)+g(s) u^{\prime}(s)\right] d s\right\} .
$$

Por indução,

$$
r(t) \leq c P_{k-1}^{-1} \exp \left\{\int_{t_{0}}^{t}\left[f(s)+g(s) u^{\prime}(s)\right] d s\right\}, \quad t \in\left[t_{0}, t_{k}\right) .
$$

Portanto, para todo $t \in J,(2.24)$ implica

$$
x(t) \leq r(t) \leq c P^{-1} \exp \left\{\int_{t_{0}}^{t}\left[f(s)+g(s) u^{\prime}(s)\right] d s\right\} .
$$

A verificação da validade da condição (2.16) pode não ser uma tarefa simples, logo é importante notar que essa condição é equivalente à seguinte:

$$
\sum_{k=1}^{\infty}\left|a_{k}\right| g\left(t_{k}\right)<\infty
$$

Este fato fica demonstrado pelo lema a seguir, que pode ser encontrado em Knopp [4].

Lema 2.8. Seja $\left(b_{k}\right)$ uma seqüência de números reais tais que $0 \leq b_{k}<1$, para $k=$ $1,2, \cdots$ Então a série $\sum_{k=1}^{\infty} b_{k}$ é convergente se e somente se o produtório $\prod_{k=1}^{\infty}\left(1-b_{k}\right)$ é positivo.

Demonstração: Mostremos primeiramente que $\sum_{k=1}^{\infty} b_{k}<\infty$ se e somente se $\prod_{k=1}^{\infty}\left(1+b_{k}\right)<\infty$. Suponhamos que $S=\sum_{k=1}^{\infty} b_{k}<\infty$. Sejam as seqüências $\left(s_{k}\right)$, $s_{k}=b_{1}+\cdots+b_{k}$, de somas parciais, e $\left(p_{k}\right)$, com $p_{k}=\left(1+b_{1}\right)\left(1+b_{2}\right) \cdots\left(1+b_{k}\right)$, de produtos parciais. Como $1+b_{k} \geq 1,\left(p_{k}\right)$ é uma seqüência crescente. Basta mostrarmos que é limitada. Notemos que, para todo $t \in \mathbb{R}, 1+t \leq \mathrm{e}^{t}$ (isto pode ser visto considerando a função $f(t)=\mathrm{e}^{t}-1-t$. Seu único ponto crítico é o zero. Como $f^{\prime \prime}(0)=1$, temos que $f$ tem um mínimo em $t=0$, e portanto $f(t) \geq 0$ ). Então, para todo $k, p_{k} \leq \mathrm{e}^{s_{n}} \leq \mathrm{e}^{S}$. Reciprocamente, temos 
$p_{k}=\left(1+b_{1}\right)\left(1+b_{2}\right) \cdots\left(1+b_{k}\right)=1+b_{1}+\cdots+b_{k}+b_{1} b_{2}+\cdots>s_{k}$. Por comparação, a convergência de $\left(p_{k}\right)$ implica que $\left(s_{k}\right)$ converge.

Consideremos novamente a equivalência entre as afirmações $S=\sum_{k=1}^{\infty} b_{k}<\infty$ e $P=\prod_{k=1}^{\infty}\left(1-b_{k}\right)>0$. Novamente, sejam as seqüências $\left(s_{k}\right)$ de somas parciais, e $\left(p_{k}\right)$, sendo desta vez $p_{k}=\left(1-b_{1}\right)\left(1-b_{2}\right) \cdots\left(1-b_{k}\right)$, de produtos parciais. Agora, $\left(p_{k}\right)$ é decrescente, por $1-b_{k} \leq 1$. Suponhamos $P>0$. Como $b_{k} \in[0,1)$, temos

$$
\left(1+b_{k}\right) \leq \frac{1}{1-b_{k}}
$$

(podemos ver claramente multiplicando a desigualdade por $\left[1-b_{k}\right]$ ). Então $p_{k}=$ $\left(1-b_{1}\right)\left(1-b_{2}\right) \cdots\left(1-b_{k}\right) \geq P>0$ nos dá $\left(1+b_{1}\right) \cdots\left(1+b_{k}\right) \leq \frac{1}{P}$. Então $S<\infty$. Reciprocamente, suponhamos $\sum_{k=1}^{\infty} b_{k}<\infty$. Então $\left(b_{k}\right)$ converge para zero. Então existe $N$ tal que $\left|b_{k}\right|<\frac{1}{2}$ para todo $k \geq N$. Não há perda de generalidade para a análise da convergência de $\left(p_{k}\right)$ se supormos $b_{k} \leq \frac{1}{2}$. Certamente, temos $\sum_{k=1}^{\infty} 2 b_{k}<\infty$. Logo, o produtório $\prod_{k=1}^{\infty}\left(1+2 b_{k}\right)=\bar{P}<\infty$. Usemos agora o fato de que, para $b_{k} \in\left[0, \frac{1}{2}\right)$,

$$
1-b_{k} \geq \frac{1}{1+2 b_{k}} .
$$

Novamente, inferimos $p_{k}=\left(1-b_{1}\right) \cdots\left(1-b_{k}\right) \geq \tilde{P}^{-1}>0$.

Agora a unicidade das soluções da equação (2.1) é fácil de ser obtida. Note que, se $u$ é do tipo $\varphi$, o mesmo ocorre com $v_{u}$. Além disso, $v_{u}$ é não decrescente em

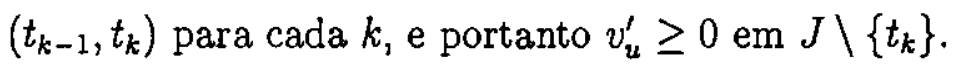

Teorema 2.9 (Unicidade de Soluções de uma EDM). Suponha que

\section{i. A função ué do tipo $\varphi$;}

ii. Existem funçōes $f, g$ em $J$ tais que

$$
|F(t, x)-F(t, y)| \leq f(t)|x-y|, \quad|G(t, x)-G(t, y)| \leq g(t)|x-y|,
$$

para todo tem $J$ e $x, y$ tais que $\left|x-x_{0}\right|,\left|y-x_{0}\right| \leq \delta$, para algum $\delta>0$;

iii. As funçōes $f, g v_{u}^{\prime}$ são integráveis em $J$; 
iv. O salto $b_{k}=v_{u}\left(t_{k}\right)-v_{u}\left(t_{k}-\right)$ satisfaz

$$
\begin{gathered}
\left|b_{k}\right| g\left(t_{k}\right)<1, \quad k=1,2, \cdots, \\
\sum_{k=1}^{\infty}\left|b_{k}\right| g\left(t_{k}\right)<\infty .
\end{gathered}
$$

Nestas condições, a equação (2.1) possui, no máximo, uma solução por $\left(t_{0}, x_{0}\right)$.

Demonstração: Se $x(\cdot)$ e $y(\cdot)$ são soluções de (2.1) por $\left(t_{0}, x_{0}\right)$, então existe $T>t_{0}$ tal que $V\left(x,\left[t_{0}, T\right]\right)<\delta$ e $V\left(y,\left[t_{0}, T\right]\right)<\delta$. Logo, $\left|x(t)-x_{0}\right|<\delta$ e $\left|y(t)-x_{0}\right|<\delta$ para todo $t \in\left[t_{0}, T\right]$.

Fazendo $z(t)=|x(t)-y(t)|$, temos, pelo Teorema 2.3,

$$
\begin{aligned}
z(t) & =\left|\int_{t_{0}}^{t}(F(s, x(s))-F(s, y(s))) d s+\int_{t_{0}}^{t}(G(s, x(s))-G(s, y(s))) d u(s)\right| \\
& \leq \int_{t_{0}}^{t} f(s) z(s) d s+\int_{t_{0}}^{t} g(s) z(s) d v_{u}(s) \\
& <\varepsilon+\int_{t_{0}}^{t} f(s) z(s) d s+\int_{t_{0}}^{t} g(s) z(s) d v_{u}(s), \quad t \in J,
\end{aligned}
$$

para todo $\varepsilon>0$.

Sendo

$$
P=\prod_{i=1}^{\infty}\left[1-\left|a_{i}\right| g\left(t_{i}\right)\right]
$$

que é não nulo pelo Lema 2.8, o Teorema 2.7 nos dá

$$
z(t) \leq \varepsilon P^{-1} \exp \left\{\int_{t_{0}}^{t}\left[f(s)+g(s) v_{u}^{\prime}(s)\right] d s\right\}, \quad t \in J .
$$

A conclusão segue da arbitrariedade de $\varepsilon>0$. 


\section{Capítulo 3}

\section{Sistemas Lineares Impulsivos}

No capítulo anterior, a equação

$$
D x=F(t, x)+G(t, x) D u
$$

i estudada principalmente como se tratando de uma perturbação impulsiva de um sistema ordinário. Veja, por exemplo, o Teorema 2.9 , onde as hipóteses sobre a parte impulsiva a caracterizam como uma perturbação. Por vezes, necessitamos considerar o sistema (3.1) como uma perturbação de um sistema que já contém impulsos. Neste capítulos, consideraremos a equação

$$
D y=A(t) y D u+f(t, y)+g(t, y) D u \quad x\left(t_{0}\right)=x_{0}
$$

como uma perturbação do sistema linear impulsivo

$$
D x=A(t) x D u, \quad x\left(t_{0}\right)=x_{0}
$$

sendo $x, y$ vetores em $\mathbb{R}^{n}, A(\cdot)$ é uma matriz real $n \times n$ contínua em $J=[0, \infty)$; $t_{0} \geq 0 ; u$ é uma função real contínua à direita de variação limitada no intervalo $J$; $f: J \times \mathbb{R}^{n} \rightarrow \mathbb{R}^{n}$ é Lebesgue-integrável e $g: J \times \mathbb{R}^{n} \rightarrow \mathbb{R}^{n}$ é integrável com respeito à medida de Borel-Stieltjes $d u$.

Para simplificarmos a análise, assumiremos $u$ do tipo $\varphi$. Mais especificamente, se $u$ tem saltos nos instantes $t_{1}, t_{2}, \cdots$, a função $u$ será assumida da forma

$$
u=t+\sum_{k=1}^{\infty} a_{k} H_{k}(t)
$$


onde $a_{k}$ é real, e estamos simplificando a notação pondo $H_{k}(t):=H_{t_{k}}(t)$, uma vez que não há risco de ambigüidade nesse procedimento. Isto é razoável porque, de modo geral, uma função contínua à direita de variação limitada contém uma parte absolutamente contínua e uma parte singular. Esta última é da forma

$$
\sum_{k=1}^{\infty} a_{k} H_{k}(t)
$$

quando as descontinuidades são isoladas. Além disso, o efeito dos impulsos sobre o sistema é visto explicitamente. Com efeito,

$$
D u=1+\sum_{k=1}^{\infty} a_{k} \delta_{k}\left(t_{k}\right)
$$

sendo $\delta_{k}$ a medida de Dirac concentrada em $t_{k}$. (Notemos que $D u \equiv 1$ em quase toda parte de $J$ ).

\section{Sistemas Lineares Impulsivos}

Assim como no caso das EDO's, a investigação das propriedades das soluções de (SIP) requer o conhecimento prévio das soluções do sistema linear (SI). Para este fim, seja $X(t)$ a matriz fundamental de

$$
\dot{x}(t)=A(t) x(t)
$$

tal que $X(0) I \mathrm{Id}$, isto é, a matriz principal em $t=0$. Dado $s \in J$, usaremos $X(t, s)=X(t) X^{-1}(s)$ para indicarmos a matriz principal em $a$ associada a $A(t)$. Então $X(t, t)=\mathrm{Id}, t \in J$. Além disso,

$$
X(t, s) X(s, r)=X(t, r), \quad t, s, r \in J
$$

e, em particular, $X(t, s)=[X(s, t)]^{-1}, \quad t, s \in J$. Seja também $B_{k}=\operatorname{Id}-a_{k} A\left(t_{k}\right)$, que é singular se e somente se $a_{k} \neq 0$ e $a_{k}^{-1}$ é autovalor de $A\left(t_{k}\right)$.

Teorema 3.1 (Solução Global de (SI)). Suponhamos que $B_{k}$ seja não singular para cada $k$. Então, para $t \in\left[t_{k-1}, t_{k}\right)$ e para todo $x_{0} \in \mathbb{R}^{n}$, a única soluçāo $x(t)$ por $\left(t_{0}, x_{0}\right)$ é dada por

$$
x(t)=X\left(t, t_{k}\right)\left[\prod_{i=1}^{k-1} B_{k-i}^{-1} X\left(t_{k-i}, t_{k-i-1}\right)\right] x_{0},
$$

sendo o produtório $\prod_{i=1}^{k-1}$ considerado como Id se $k=1$. 
Demonstração: Primeiramente, consideremos $t \in\left[t_{0}, t_{1}\right)$. Então $u(t)=t \mathrm{e}$, pelo Teorema 2.3, temos que

$$
x(t)=X\left(t, t_{0}\right) x_{0}
$$

Em $t=t_{1}$, sabemos que

$$
\begin{aligned}
x\left(t_{1}\right) & =x_{0}+\int_{t_{0}}^{t_{1}} A(s) x(s) d u(s) \\
& =x\left(t_{1}-\varepsilon\right)+\int_{t_{1}-\varepsilon}^{t_{1}} A(s) x(s) d u(s),
\end{aligned}
$$

$\operatorname{com} \varepsilon>0$ suficientemente pequeno. Fazendo $\varepsilon \rightarrow 0+$ e usando o fato de que $X(\cdot, s)$ é contínuo, obtemos

$$
\begin{aligned}
x\left(t_{1}\right) & =\lim _{\varepsilon \rightarrow 0+}\left[x\left(t_{1}-\varepsilon\right)+\int_{t_{1}-\varepsilon}^{t_{1}} A(s) x(s) d u(s)\right] \\
& =X\left(t_{1}, t_{0}\right) x_{0}+a_{1} A\left(t_{1}\right) x\left(t_{1}\right)
\end{aligned}
$$

que nos dá

$$
B_{1} x\left(t_{1}\right)=X\left(t_{1}, t_{0}\right) x_{0}
$$

Por $B_{1}$ ser invertível, segue

$$
x\left(t_{1}\right)=B_{1}^{-1} X\left(t_{1}, t_{0}\right) x_{0}
$$

Para $t \in\left[t_{1}, t_{2}\right)$, temos que $x(t)=X\left(t, t_{1}\right) x\left(t_{1}\right)$, sendo $x\left(t_{1}\right)$ dado por (3.7). Argumentando analogamente, segue que $x\left(t_{2}\right)=X\left(t_{2}, t_{1}\right) x\left(t_{1}\right)+a_{2} A\left(t_{2}\right) x\left(t_{2}\right)$, que equivale a

$$
B_{2} x\left(t_{2}\right)=X\left(t_{2}, t_{1}\right) x\left(t_{1}\right)
$$

Esta equação é resolvida por

$$
x\left(t_{2}\right)=B_{2}^{-1} X\left(t_{2}, t_{1}\right) B_{1}^{-1} X\left(t_{1}, t_{0}\right) x_{0},
$$

que é a fórmula em (3.5) para $k=3$ e $t=t_{2}$. O caso para $k$ geral segue por indução.

A unicidade da solução dada em (3.5) vem da escolha única de $x\left(t_{k}\right)$ que, como em (3.6) e (3.8), satisfaz a $B_{k} x\left(t_{k}\right)=X\left(t_{k}, t_{k-1}\right) x\left(t_{k-1}\right)$, e à maneira única de se estender a solução em $\left[t_{k}, t_{k+1}\right)$. 
Corolário 3.2 (Solução Global de (SI) com Coeficientes Constantes). Seja $A(t)=A$ uma matriz constante e suponhamos que $B_{k}$ seja invertivel para cada $k$. Neste caso, $X(t, s)=\mathrm{e}^{(t-s) A}$. Para detalhes sobre o operador $\mathrm{e}^{t A}$, veja Hale [2, Chap. III.4], por exemplo. Sabemos que $\mathrm{e}^{s A}$ é expresso por uma série de potências em A. Então $e^{\left(t_{k}-t_{k-1}\right) A}$ e $B_{j}^{-1}$ comutam, por seus inversos comutarem. Neste caso, (3.5) assume a forma mais simples

$$
x(t)=\left[\prod_{i=1}^{k-1} B_{k-i}^{-1}\right] \mathrm{e}^{\left(t-t_{0}\right) A} x_{0}, \quad t \in\left[t_{k-1}, t_{k}\right) .
$$

Observação 3.3. Se $a_{k}=0$ para todo $k$, caso no qual $B_{k}=\mathrm{Id}$, entāo, por (3.4), $a$ fórmula em (3.5) fica reduzida a

$$
x(t)=X\left(t, t_{0}\right) x_{0},
$$

que é a soluçāo do problema clássico

$$
\dot{x}(t)=A(t) x(t), \quad x\left(t_{0}\right)=x_{0}
$$

ao qual (SI) se reduz nas condições anteriores.

O teorema a seguir trata do problema de continuarmos soluções em algum $t_{k}$ quando $B_{k}$ é singular. No Teorema 3.1 , o fato de $B_{k}$ ser não singular foi usado apenas quando buscávamos um valor $x\left(t_{k}\right)$ tal que

$$
B_{k} x\left(t_{k}\right)=X\left(t_{k}, t_{k-1}\right) x\left(t_{k-1}\right),
$$

(veja (3.6) e (3.8)) e portanto pudemos continuar a solução até $t_{k+1}$. Bastou aplicar $B_{k}^{-1}$ aos membros de (3.9). Em casos nos quais esse procedimento não é possível, podemos utilizar um conhecido resultado de álgebra linear, enunciado no lema abaixo, que nos dá uma caracterização de quando é possível resolvermos em $x$ um sistema da forma $A x=b$, com $A$ uma matriz $n \times n$, e $x, b$ vetores coluna.

Lema 3.4. $O$ sistema $A x=b$ com incógnita $x$, sendo $A$ uma matriz real $n \times n$ e $b$ um vetor coluna em $\mathbb{R}^{n}$, admite solução se e somente se b é ortogonal ao núcleo de $A^{\mathrm{T}}$, ou seja, $\langle b, y\rangle=0$ para todo y tal que $A^{\mathrm{T}} y=0$.

Teorema 3.5 (Extensão de Soluções de (SI) $\operatorname{com} B_{k}$ Singular). Suponha que $x(t)$ seja solução de (SI) para t no intervalo $\left[t_{0}, t_{k}\right)$. Então esta solução pode ser estendida para $\left[t_{0}, t_{k}\right]$ (e portanto para $\left.\left[t_{0}, t_{k+1}\right\rangle\right)$ se e somente se vale uma das seguintes alternativas: 1) $B_{k}$ é não singular ou 2) $X\left(t_{k}, t_{k-1}\right) x\left(t_{k-1}\right)$ é nulo ou é autovetor de $A\left(t_{k}\right)^{\mathrm{T}}$ associado ao autovalor $a_{k}^{-1}$. 
Demonstração: $O$ caso no qual $B_{k}$ é não singular tem tratamento análogo ao que foi feito na demonstração do Teorema 3.1.

Se $B_{k}$ é singular, então $a_{k} \neq 0$, caso contrário $B_{k}=\mathrm{Id}$. Neste caso, $a_{k}^{-1}$ é autovalor de $A\left(t_{k}\right)$, portanto também de $A\left(t_{k}\right)^{\mathrm{T}}$. Notemos que $v$ anula $B_{k}^{\mathrm{T}}$ se e somente se $v=0$ ou $v$ é autovetor de $A\left(t_{k}\right)^{\mathrm{T}}$ associado a $a_{k}^{-1}$. Assim, o Lema $3.4 \mathrm{e}$ as considerações anteriores a este Teorema, (3.9) admitirá solução $x\left(t_{k}\right)$ se e somente se $X\left(t_{k}, t_{k-1}\right) x\left(t_{k-1}\right)$ é ortogonal a todo autovetor de $A\left(t_{k}\right)^{\mathrm{T}}$ associado ao autovalor $a_{k}^{-1}$.

Observação 3.6. Suponhamos que $B_{i}, i=1,2, \cdots, j-1$ seja nāo singular, $a_{j}^{-1}$ seja autovalor de $A\left(t_{j}\right)$ e $x_{0}=0$. O fato de ser $x_{0}=0$ implica $x(t)=0$ para $t \in\left[t_{0}, t_{j}\right)$, pela função nula ser solução de (SI) neste intervalo e pela unicidade da solução nos intervalos $\left[t_{n-1}, t_{n}\right)$ por ser $B_{i}$ não singular, $i=1,2, \cdots, j-1$. Então $X\left(t_{j}, t_{j-1}\right) x_{j-1}=$ 0 , sendo portanto ortogonal ao núcleo de $B_{j}^{\mathrm{T}}$. Para resolvermos (3.9), basta escolhermos qualquer elemento $x\left(t_{j}\right)$ em $\operatorname{ker} B_{j} \neq\{0\}$, que é o auto-espaço gerado pelo autovalor $a_{j}^{-1}$ com relação a $A\left(t_{j}\right)$. Isto nos dá que (SI) admite infinitas soluções em $\left[t_{0}, t_{j+1}\right)$ com a condição inicial $x\left(t_{0}\right)=0$.

Exemplo 3.7. Seja $J=[0, \infty)$. Considere o sistema (SI), com

$$
A(t)=A=\left[\begin{array}{ll}
2 & 1 \\
3 & 4
\end{array}\right]
$$

Em (3.2), tomemos $t_{k}=k, k \in \mathbb{N} ; a_{1}=-1, a_{2}=1$ e $a_{k}=k, k \geq 3$. A matriz A tem 1 e 5 como autovalores. Logo, apena $a_{2}^{-1}$ é autovalor de $A$ e $A^{\mathrm{T}}$. Escolhamos $x_{0}=\left(\frac{1}{3}\right) . O$ Teorema 3.1 garante a existência de uma única solução de (SI) por $\left(0, x_{0}\right)$ em $\left[t_{0}, t_{2}\right)$. De fato, não é difícil ver que esta solução é dada por $x(t)=\left(\begin{array}{l}x_{1}(t) \\ x_{2}(t)\end{array}\right)$, com

$$
x_{1}(t)=\left\{\begin{array}{ll}
\mathrm{e}^{5 t} & \text { para } 0 \leq t<1 \\
\frac{1}{6} \mathrm{e}^{5 t} & \text { para } 1 \leq t<2
\end{array} \quad \text { e } x_{2}(t)= \begin{cases}3 \mathrm{e}^{5 t} & \text { para } 0 \leq t<1 \\
\frac{1}{2} \mathrm{e}^{5 t} & \text { para } 1 \leq t<2\end{cases}\right.
$$

O espaço dos autovetores de $A^{\mathrm{T}}$ associados a 1 é gerado por $v_{1}=\left(\begin{array}{c}-3 \\ 1\end{array}\right)$. Como $x\left(t_{2}-\right)=$ $\frac{1}{2} \mathrm{e}^{10}\left(\begin{array}{c}1 / 3 \\ 1\end{array}\right)$ é ortogonal a $v_{1}$, o sistema (3.9) admite soluçāo. Fazendo os cálculos, $x\left(t_{k}\right)$ resolve (3.9) se e somente se

$$
x\left(t_{k}\right)=\frac{1}{2} \mathrm{e}^{10}\left(\begin{array}{c}
\lambda \\
-\lambda-\frac{1}{3}
\end{array}\right), \quad \lambda \in \mathbb{R} .
$$

Para $k \geq 3, B_{k}$ é nāo singular, e a extensāo é feita de maneira única a partir de então. 
Exemplo 3.8 (Problema do Crescimento). O crescimento de uma cultura de bactérias em medicina, decaimento da massa de elementos radioativos em física, crescimento da populaçāo e da poluição, etc, são alguns dos problemas modelados por equaçōes diferenciais do tipo "evolução".

Seja $x(t)$ uma quantidade considerada e assumamos que sua taxa de crescimento seja proporcional a $x(t)$, para cada $t$. Este problema é modelado pela equação diferencial ordinária

$$
\dot{x}(t)=\alpha x(t), \quad x\left(t_{0}\right)=x_{0},
$$

sendo $\alpha$ a constante de proporcionalidade. A única solução para este problema é dada por

$$
x(t)=x_{0} \mathrm{e}^{\alpha\left(t-t_{0}\right)}, \quad t \geq t_{0} .
$$

Estudemos uma nova situação na qual este modelo necessita ser modificado. Considere um lago para criação de peixes. Assumiremos que a taxa de crescimento, em cada instante, é proporcional à quantidade $x(t)$ de peixes no lago (Lei de Malthus). Em determinados instantes de tempo $t_{1}, t_{2}, \cdots$, é retirada do lago uma quantidade de peixes, com uma rede. O comportamento da população de peixes é, portanto, impulsivo, com impulsos nos instantes $t_{1}, t_{2}, \cdots$. Também, a quantidade de peixes retirada do lago depende da densidade de peixes nos instantes $t_{i}$.

Este problema pode ser satisfatoriamente resolvido pela equação

$$
D x(t)=\alpha x(t) D u, \quad x\left(t_{0}\right)=x_{0},
$$

sendo u dada por

$$
u(t)=t+\sum_{k=1}^{\infty} \frac{a_{k}}{\alpha+\alpha a_{k}} H_{k}(t),
$$

com $H_{k}$ definida por $H_{k}(t)=H\left(t-t_{k}\right)$, e $a_{k}$ são números reais. Note que as descontinuidades de u são isoladas e devemos ter $a_{k} \neq-1$. Temos, portanto,

$$
D u=1+\sum_{k=1}^{\infty} \frac{a_{k}}{\alpha+\alpha a_{k}} \delta_{t_{k}} .
$$

As hipoteses do Corolário 3.2 estão satisfeitas, dando-nos que a única solução de (3.10) é expressa por

$$
x(t)=\left[\prod_{i=1}^{k}\left(1+a_{i}\right)\right] \mathrm{e}^{\left(t-t_{0}\right) \alpha} x_{0}, \quad \text { para } t \in\left[t_{k}, t_{k+1}\right) .
$$


Desta forma, o salto da solução nos instantes $t_{k}$ é dado por

$$
x\left(t_{k}+\right)-x\left(t_{k}-\right)=a_{k}\left[\prod_{i=1}^{k}\left(1+a_{i}\right)\right] \mathrm{e}^{\left(t-t_{0}\right) \alpha} x_{0}=a_{k} x\left(t_{k}-\right) .
$$

Em situações particulares como essa, a teoria apenas fornece orientaçāo de procedimentos a serem adotados, visto que seu uso pode envolver relevantes problemas de adaptação dos resultados gerais no estabelecimento de sua aplicabilidade.

\section{Fórmula da Variação das Constantes}

A fórmula da variação das constantes é um importante recurso utilizado no estudo das propriedades de sistemas de equações diferenciais ordinárias lineares com pequenas perturbações. Para o mesmo fim, a teoria das equações em medida possui uma versão desse resultado, obtida acrescentando-se novos termos à antiga fórmula, devido ao comportamento impulsivo das soluções nos instantes $t_{k}$. Para simplificarmos a análise, abordaremos apenas o caso no qual as transformaçōes $B_{k}$, introduzidas na página 48 , são não singulares para todo $k$. O caso geral pode ser obtido a partir do que será feito no Teorema 3.10, bastando algumas alterações na mesma linha do que foi feito na demonstração do Teorema 3.5. Começaremos pela versão da fórmula para equações ordinárias.

Teorema 3.9 (Fórmula da Variação das Constantes para EDO). Sejam

$$
\begin{gathered}
\dot{y}=A(t) y+h(t) \\
\dot{x}=A(t) x,
\end{gathered}
$$

sistemas lineares de equações diferenciais ordinárias não homogêneo e homogêneo, respectivamente. Se $X(t)$ é uma matriz fundamental de (3.12), então toda solução $y(t)$ de (3.11) é dada pela formula

$$
y(t)=X(t)\left[X^{-1}(\tau) x(\tau)+\int_{\tau}^{t} X^{-1}(s) h(s) d s\right]
$$

para todo número real $\tau$. 
Demonstração: Seja $y(t)$ solução de (3.11). Como $X(t)$ é não singular para cada $t$, segue que a mudança de variáveis $y=X(t) x$ define um homeomorfismo de $\mathbb{R}^{n} \mathrm{em}$ $\mathbb{R}^{n}$ para cada $t$. Como $A(t)=X^{-1}(t) \dot{X}(t)$, então

$$
\dot{x}=\frac{d}{d t}\left[X^{-1} y\right]=X^{-1}(A y+h)-X^{-1} A y=X^{-1} h .
$$

Isto implica

$$
x(t)=X^{-1}(\tau) y(\tau)+\int_{\tau}^{t} X^{-1}(s) h(s) d s
$$

por ser $x(\tau)=X^{-1}(\tau) y(\tau)$. Aplicando $X(t)$ aos membros da equação (3.14), obtemos a relação (3.13).

A razão pela qual a equação em (3.13) é chamada fórmula da variação das constantes é motivada por toda solução ser da forma $y(t)=X(t) c(t)$, sendo $c(t)$ o termo entre colchetes na equação (3.13), e por $c(t)$ ser constante no caso homogêneo.

Considerando novamente a matriz principal em $s \quad X(t, s)$, podemos reescrever a equaçāo (3.13) na forma mais simples

$$
y(t)=X(t, \tau) y(\tau)+\int_{\tau}^{t} X(t, s) h(s) d s
$$

Teorema 3.10 (Fórmula da Variação das Constantes para EDM). Suponhamos $B_{k}$ invertivel para cada $k=1,2, \cdots$. Se $x(t)$, dado por (3.5), é uma soluçāo do sistema linear (SI) então, para t no intervalo $\left[t_{k-1}, t_{k}\right)$, toda solução $y(t)$ de (SIP) é dada por

$$
\begin{aligned}
y(t)= & x(t)+\int_{t_{0}}^{t} X(t, s) f(s, y(s)) d s+\int_{t_{0}}^{t} X(t, s) g(s, y(s)) d u(s) \\
& +X\left(t, t_{k-1}\right) \sum_{i=1}^{k-1} a_{i} X\left(t, t_{k-1}\right) B_{k-1}^{-1}\left[\prod_{j=i}^{k-1} X\left(t_{k-j+1}, t_{k-j}\right) B_{k-j}^{-1}\right] A\left(t_{i}\right)\left(F_{i}+G_{i}\right)
\end{aligned}
$$

sendo
$F_{i}=\int_{t_{0}}^{t_{i}} X\left(t_{i}, s\right) f(s, y(s)) d s, \quad G_{i}=\int_{t_{0}}^{t_{i}} X\left(t_{i}, s\right) g(s, y(s)) d u(s), i=1,2, \cdots, k-1$. 
Demonstração: Para $t \in\left[t_{0}, t_{1}\right)$, o Teorema 3.9 implica

$$
y(t)=X\left(t, t_{0}\right) x_{0}+\int_{t_{0}}^{t} X(t, s) f(s, y(s)) d s+\int_{t_{0}}^{t} X(t, s) g(s, y(s)) d s
$$

Como na prova do Teorema 3.1,

$$
y\left(t_{1}\right)=y\left(t_{1}-h\right)+\int_{t_{1}-h}^{t_{1}} A(s) y(s) d u(s)+\int_{t_{1}-h}^{t_{1}} f(s, y(s)) d s+\int_{t_{1}-h}^{t_{1}} g(s, y(s)) d u(s)
$$
de De (3.16), fazendo $h \rightarrow 0+$, obtemos I|

$$
\begin{aligned}
\begin{aligned}
y\left(t_{1}\right)= & X\left(t_{1}, t_{0}\right) x_{0}+\int_{t_{0}}^{t_{1}} X\left(t_{1}, s\right) f(s, y(s)) d s \\
& \left.+\int_{\left[t_{0}, t_{1}\right)} X\left(t_{1}, s\right) g(s, y(s)) d u(s)+d_{1} A\left(t_{1}\right) y\left(t_{1}\right)\right]+a_{1} g\left(t_{1}, y\left(t_{1}\right)\right)
\end{aligned}
\end{aligned}
$$

que, por $B_{1}$ ser invertível, nos dá

$$
\begin{aligned}
& y\left(t_{1}\right)=B_{1}^{-1}\left[X\left(t_{1}, t_{0}\right) x_{0}+F_{1}+\int_{\left[t_{0}, t_{1}\right)} X\left(t_{1}, s\right) g(s, y(s)) d u(d)+a_{1} g\left(t_{1}, y\left(t_{1}\right)\right)\right] \\
& X\left(t_{1}, t_{1}\right)=\mathrm{Id}
\end{aligned}
$$

$$
\begin{aligned}
& a_{1} g\left(t_{1}, y\left(t_{1}\right)\right)=a_{1} X\left(t_{1}, t_{1}\right) g\left(t_{1}, y\left(t_{1}\right)\right)=\int_{\left\{t_{1}\right\}} X\left(t t_{1}, s\right) g(s, y(s)) d u(s) . \\
& B_{1}^{-1} X\left(t_{1}, t_{0}\right) x_{0}=x\left(t_{1}\right), \text { de }
\end{aligned}
$$

temos

$$
B_{1}^{-1}=B_{1}^{-1}\left[\left(\mathrm{Id}-a_{1} A\left(t_{1}\right)\right)+a_{1} A\left(t_{1}\right)\right]=\mathrm{Id}+a_{1} B_{1}^{-1} A\left(t_{1}\right),
$$

$$
\begin{gathered}
y\left(t_{1}\right)=x\left(t_{1}\right)+B_{1}^{-1}\left(F_{1}+G_{1}\right) \\
=x\left(t_{1}\right)+F_{1}+G_{1}+a_{1} B_{1}^{-1} A\left(t_{1}\right)\left(F_{1}+G_{1}\right) . \\
\text { Para } t \in\left[t_{1}, t_{2}\right) \text {, sabemos } \\
y(t)=X\left(t, t_{1}\right) y\left(t_{1}\right)+\int_{t_{1}}^{t} X(t, s) f(s, y(s)) d s+\int_{t_{1}}^{t} X(t, s) g(s, y(s)) d s, \\
\text { com } y\left(t_{1}\right) \text { dado por (3.17). Da equação anterior, de } x(t)=X\left(t, t_{1}\right) x\left(t_{1}\right) \text { para } t \in \\
{\left[t_{1}, t_{2}\right)(\text { ver }(3.5)) \text { e de }} \\
X\left(t, t_{1}\right)\left(F_{1}+G_{1}\right)=\int_{t_{0}}^{t_{1}} X(t, s) f(s, y(s)) d s+\int_{t_{0}}^{t_{1}} X(t, s) g(s, y(s)) d u(s),
\end{gathered}
$$


tiramos

$$
\begin{aligned}
y(t)= & X\left(t, t_{1}\right)\left[x\left(t_{1}\right)+F_{1}+G_{1}+a_{1} B_{1}^{-1} A\left(t_{1}\right)\left(F_{1}+G_{1}\right)\right] \\
& +\int_{t_{1}}^{t} X(t, s) f(s, y(s)) d s+\int_{t_{1}}^{t} X(t, s) g(s, y(s)) d u(s) \\
= & x(t)+\int_{t_{0}}^{t} X(t, s) f(s, y(s)) d s+\int_{t_{0}}^{t} X(t, s) g(s, y(s)) d u(s) \\
& +a_{1} X\left(t, t_{1}\right) B_{1}^{-1} A\left(t_{1}\right)\left[F_{1}+G_{1}\right], \quad t \in\left[t_{1}, t_{2}\right) .
\end{aligned}
$$

Como anteriormente, podemos mostrar

$$
\begin{aligned}
y\left(t_{2}\right)= & x\left(t_{2}\right)+F_{2}+G_{2}+a_{2} B_{2}^{-1} A\left(t_{2}\right)\left(F_{2}+G_{2}\right) \\
& +a_{1} B_{2}^{-1} X\left(t_{2}, t_{1}\right) B_{1}^{-1} A\left(t_{1}\right)\left(F_{1}+G_{1}\right) .
\end{aligned}
$$

Novamente, para $t \in\left[t_{2}, t_{3}\right)$,

$$
\begin{aligned}
y(t)= & X\left(t, t_{2}\right)\left[x\left(t_{1}\right)+F_{2}+G_{2}\right. \\
& \left.+a_{2} B_{2}^{-1} A\left(t_{2}\right)\left(F_{2}+G_{2}\right)+a_{1} B_{2}^{-1} X\left(t_{2}, t_{1}\right) B_{1}^{-1} A\left(t_{1}\right)\left(F_{1}+G_{1}\right)\right] \\
& +\int_{t_{2}}^{t} X(t, s) f(s, y(s)) d s+\int_{t_{2}}^{t} X(t, s) g(s, y(s)) d u(s) \\
= & x(t)+\int_{t_{0}}^{t} X(t, s) f(s, y(s)) d s+\int_{t_{0}}^{t} X(t, s) g(s, y(s)) d u(s) \\
& +a_{2} B_{2}^{-1} A\left(t_{2}\right)\left(F_{2}+G_{2}\right)+a_{1} X\left(t, t_{1}\right) B_{1}^{-1} A\left(t_{1}\right)\left[F_{1}+G_{1}\right], \quad t \in\left[t_{2}, t_{3}\right) .
\end{aligned}
$$

O caso geral, dado por (3.15), é obtido por aplicações sucessivas desses argumentos. 


\section{Referências Bibliográficas}

[1] Graves, L. "Theory of Functions of Real Variables”, McGraw-Hill, N. York (1946).

[2] Hale, J. K. “Ordinary Differential Equations”, Robert Krieger Publishing Company, N. York (1980).

[3] Hounie, J. "Teoria Elementar das Distribuições", $12^{\circ}$ Colóquio Brasileiro de matemática, IMPA, Rio de Janeiro (1979).

[4] Knopp, K. "Theory and Application of Infinite Series", Blackie \& Son Limited, London and Glasgow, (1951).

[5] Pandit, S. G. \& Deo, S. G., "Differencial Systems Involving Impulses”, SpringerVerlag, N. York (1982).

[6] Royden, H. L. "Real Analysis”, Collier-Macmillan Canada, Ltd., Toronto, Ontario (1968).

[7] Rudin, W. "Princípios de Análise Matemática", Ao Livro Técnico S.A. \& Editora UnB, Rio de Janeiro (1971). 


\section{Índice Remissivo}

$\mathcal{B}(\sigma$-álgebra de Borel), 7, 9

$B V$ (funções de variação limitada), 21

$\bigodot_{c}^{\infty}$ (funções $\complement^{\infty}$ de suporte compacto), 28

Conjunto

boreliano, 7,9

mensurável, 5, 6, 11

positivo, negativo, nulo, 8,9

Continuidade absoluta, 26

$\mathcal{D}$ (espaço das distribuições), 28

$\Delta \alpha_{i}, 12$

$\delta$ (delta de Dirac), 28

Decomposição de Hahn, 9

Derivada

de Dini, 20

distribucional, 30

$d f$ (medida gerada por $f$ ), 10

Diferenciabilidade, 20

à direita, à esquerda, 20

Distribuição, 28

Equação diferencial em medida, 33

existência local de soluções, 38

representação integral, 35

sistema linear impulsivo, 48

solução de uma, 33

unicidade de soluções, 45
Fórmula da variação das constantes, 53,54

\section{Função}

absolutamente contínua, 26, 30

característica, 16

crescente, 10, 11, 24

de Heaviside, 31

de variação limitada, 21, 31

diferenciável, 20

à direita, à esquerda, 20

do tipo $\varphi, 27$

integrável, 17

localmente integrável, 29, 30

medida exterior $\left(m^{*}\right), 6$

mensurável, 15, 18

Riemann-integrável, 12, 18

simples, 16

variação positiva, negativa, 22

variação total $\left(v_{f}\right), 21$

Identificação, 29, 30

Integração por partes, 14, 23

Integral

de função em $\mathbb{R}^{n}, 19$

de funções simples, 16

de Lebesgue, 17

de Lebesgue-Stieltjes, 17

de Riemann, 12 
de Riemann-Stieltjes, 12, 22

indefinida, 18, 27

Lema de Gronwall

para EDM, 41

para EDO, 41

$\mathfrak{M}$ (conjuntos Lebesgue-mensuráveis), 7

$m$ (medida de Lebesgue), 7

$m^{*}$ (medida exterior), 6, 7

Medida, 5, 8

com sinal, 8,11

completa, 5

de Borel, 9, 10

de Borel-Stieltjes, 10, 11

de Lebesgue, 7, 11, 17

espaço de, 5

exterior, 6

finita, 5

gerada por função, 10

positiva, 5

variação total, 9

Número real estendido $(\overline{\mathrm{R}}), 4$

Princípio da seleção de Helly, 25

Quase toda parte, 3

$\overline{\mathrm{R}}$ (número real estendido), 4

$\Re$ (funções Riemann-integráveis), 12

Representação integral de EDM, 35

$\mathrm{R} \int$ (integral de Riemann), 12

$\sigma$-álgebra, 5, 9

de Borel, 9
Sistema linear impulsivo (SI), 47 perturbado (SIP), 47

Solução de EDM, 33

Suporte, 28

$\langle T, \phi\rangle, 28$

Teorema

convergência dominada de Lebesgue, 19

decomposição de Hahn, 9

diferenciabilidade

funções crescentes, 21

funções de variação limitada, 22

existência local de soluções, 38

fórmula da variação das constantes, 54

integração por partes, 14, 23

lema de Gronwall para EDM, 41

representação integral de uma EDM,

35

solução global para EDM linear, 48

unicidade de soluções de uma EDM,

45

Tipo $\varphi, 27$

$V(f,[a, b])$ (variação total), 21

Variação limitada, 21

Variação total, 21

$v_{f}$ (função variação total de $f$ ), 21 